\title{
WIEDERHOLUNG UND DIFFERENZ
}

\section{Beobachtungen zum liniengenauen Motivtransfer in der spätmittelalterlichen Buchmalerei}

Echte Epochengrenzen, Zäsuren, gibt es selten. Unser modernes Verständnis des Begriffs Kopie, mit all seinen problematischen Implikationen, hat sich wohl erst in der Mitte des 16. Jahrhunderts durchgesetzt. Viel stärker als bislang von der Kunstgeschichtsschreibung thematisiert, vollzog sich mit der dichotomischen Trennung zwischen Original und Kopie ein systematischer Bruch, dessen Nachwirkungen bis in unsere Zeit reichen. Trotz erheblicher Widerstände seitens anti-dialektischer Strömungen des Poststrukturalismus und künstlerischer Bemühungen etwa aus dem Umfeld der Appropriation Art gilt die Klassifizierung eines Kunstwerks als Original nicht nur auf dem Kunstmarkt, sondern auch in den Museen und Universitäten weiterhin als ein zentrales Bewertungskriterium. Parallel dazu konnte die historisch belastete Kopie den ihr entgegengebrachten Vorbehalt ontischer Nachrangigkeit bis heute nicht abschütteln.

Zu Beginn des 15. Jahrhunderts war das Verhältnis von Auftraggebern und Künstlern zum Kopieren älterer Werke noch weitgehend ungetrübt, es machte sogar einen nicht unwesentlichen Teil der Kunstproduktion aus. Im Frühjahr 1402 beauftragte etwa der burgundische Herzog Philipp der Kühne die noch jungen, aus der berühmten Malerfamilie Maelwael („Mal schön“) stammenden Brüder Limburg damit, "[...] die Miniaturen einer sehr schönen und wertvollen Bibel zu vollenden, die der besagte Herr [Philipp der Kühne] kurz zuvor beginnen ließ [...]“. ${ }^{1}$ Dieser in den burgundischen Rechnungsbüchern dokumentierte Auftrag wurde zuerst von Paul Durrieu ${ }^{2}$ mit zwei in der Pariser Bibliothèque Nationale aufbewahrten Exemplaren ${ }^{3}$ einer besonders bilderreichen

1 Frz. Wortlaut nach Gorissen 1954, 203 f.: „[...] parfaire les histoires d'une tres belle et notable Bible que avoit nagaires fait encommencier ledit seigneur“. ÜS: Dückers 2005, 94 f.; Cf. Gorissen 1954 153-221; Meiss 1974, 72; Dückers 2005, 85-95.

2 Durrieu 1895, 120-122.

3 Paris, Bibliothèque nationale, ms. fr. 167 sowie Paris, Bibliothèque nationale, ms. fr. 166, cf. z. B Lowden 2000, vol. 1, 221-250, 251-284; Dückers 2009, 85-95; König 2010, 261-305; Husband 2012, 43 f. Vollständige Digitalisate beider Handschriften sind auf Gallica verfügbar: a) Français 167: http://gallica.bnf.fr/ark:/12148/btv1b8447300c (letzter Zugriff 21.04.2015); b) Français 166: http://gallica.bnf.fr/ark:/12148/btv1b90590452 (letzter Zugriff 21.04.2015). 
Bibelvariante, der Bible moralisée, in Verbindung gebracht. Obgleich Durrieus Identifikation nicht unwidersprochen blieb und vom Autor selbst nur unter Vorbehalt vorgeschlagen worden war, ${ }^{4}$ steht der enge Bezug zwischen den beiden angesprochenen Handschriften auch unabhängig von der erwähnten Quelle außer Frage. Die 1349-1352 in der Nachfolge des Buchmalers Jean Pucelle für Johann den Guten ${ }^{5}$ entstandene Bible moralisée (Français 167) muss den Brüdern Limburg gut fünfzig Jahre später zur Ausmalung des von ihnen begonnenen Exemplars (Français 166) als exemplum ${ }^{6}$ vorgelegen haben.

Dabei war es offenbar erwünscht, dass das Seitenlayout, die Schrift und der Schriftdekor der älteren Handschrift in nahezu faksimilierender Weise vom Schreiberatelier wiederholt wurden: Die noch sehr jungen Buchmaler übernahmen in den drei vollständig von ihnen illuminierten Lagen für die Rahmung der Bilder das System des alternierenden Wechsels zwischen Sechspass und Hochrechteck und kopierten oft wörtlich die älteren Motive. ${ }^{7}$ Anders als die Schreiber wichen sie aber in entscheidenden Punkten, etwa in der Darstellung von Körpern, Räumen und Landschaften von ihrer Vorlage ab und gestatteten sich die Freiheit zur Entfaltung eines eigenen Stilidioms. Diese Art des Kopierens kann als typisch für die mittelalterliche Kunstproduktion insgesamt angesehen werden: Die Abhängigkeit zur Vorlage mag erwünscht, sogar vorgegeben gewesen sein, zu einer stilgetreuen Wiedergabe des exemplums kam es dagegen in der Regel nicht. ${ }^{8}$ Warum Philipp der Kühne überhaupt die Kopie (Français 166) einer in seinem Besitz befindlichen Handschrift (Français 167) anfertigen ließ, bleibt indes rätselhaft. Verführerisch, doch allzu kühn erscheint der Gedanke, dass ihn vornehmlich die Aktualisierung, die (stilistische) Differenz der neuen Handschrift im Vergleich zur älteren, interessierte. In welchem Maße die womöglich großzügig für diesen Auftrag entlohnten Brüder Limburg ${ }^{9}$ einer noch bis in das Jahr 1518 der Handschrift beigefügten, auf immerhin zwei Lagen (i.d.R. zw. 12-16 Blatt) ausgedehnten Anweisung zur Ausführung der Miniaturen folgten, kann nicht mehr beantwortet werden. Sie ist bedauerlicherweise verloren gegangen. Die umfangreichen Instruktionen belegen aber, dass das Kopieren sorgsam Schritt für Schritt vorbereitet worden war. ${ }^{10}$

4 Beispielsweise durch Sterling 1987, 150-155; Colenbrander 1991, 109-116; Dückers 2009, 85-95; König 2010, 261-266.

5 Avril unterscheidet im bilderreichen Kodex fünfzehn unterschiedliche Maler in zwei größeren Stilgruppen voneinander, cf. Avril 1972, 100-114.

6 Zum Begriff des exemplum, cf. Scheller 1995, 9-18.

7 Zur Genese der Handschrift, cf. König 1982, 188 f.; Lowden 2000, vol. 1, 221-250, 251-284; König 2010, 261-305.

8 Cf. dazu auch die Überlegungen bei Alexander 1992, 72 f., 76 f., 100 f., 105-107, 121 f., 125 f., 130 f., $135,138$.

9 König fasst den aktuellen Kenntnisstand am übersichtlichsten zusammen und meldet berechtigte Zweifel an, dass die Bezahlung der Brüder Limburg für nur drei vollständig ausgemalte Lagen kaum mit den in den Quellen genannten Auszahlungen von zweimal 600 Francs sowie Sonderzahlungen übereinzubringen sei, cf. König 2010, 261-266.

10 Cf. Meiss 1974, 82; Durrieu 1895, 121; Delisle 1890, 243 und Lowden 2000, vol. 1, 255: „[...] avec deux cayés de papier ou sont contenus la forme de faire les dictz histoires.“ („[...] mit zwei Lagen 


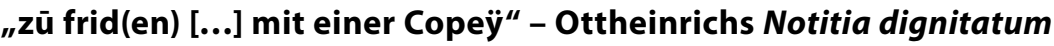

Als gut hundertfünfzig Jahre später Pfalzgraf Ottheinrich schrieb, er sei „zū frid(en) [...] mit einer Copeÿ",11 ist das deutlich als zähneknirschendes Zugeständnis zu verstehen, denn eigentlich begehrte er ein "Original“, den Codex spirensis, den ihm die Speyerer Domherren aber weder verkaufen noch ausleihen wollten. ${ }^{12}$ Dass der Wert des Originals - in diesem Fall handelt es sich um die ottonische Abschrift eines römischen Staatsrechtsbuches aus dem mittleren 5. Jahrhundert - wohl besonders in seiner Historizität bestand, wird in einem weiteren Brief Ottheinrichs vom 20. März 1550 deutlich, in dem er darauf dringt, die Notitia dignitatum "uf die alt handt malen zu lassen“. ${ }^{13}$ Als ihm die Domherren dann tatsächlich eine Abschrift zusandten, die zwar vollständig die Texte und Bilder des Codex spirensis wiederholte, in der die Bilder jedoch in der Manier des 16. Jahrhunderts von einem Speyerer Buchmaler ausgeführt worden waren, drängte der Pfalzgraf enttäuscht darauf, seine eigenen Buchmaler schicken zu dürfen, damit diese "uf geol-drenckt pappeir die alte figuren durch zeichnen“ konnten. ${ }^{14}$ Auf Grundlage jener linien- und farbgenauen Pausen auf Papier wurden alle Miniaturen der Notitia dignitatum möglichst exakt auf neue Pergamentbögen übertragen und schließlich Ottheinrichs erstem Exemplar angefügt. Das Ergebnis, ein heute in der Münchner Staatsbibliothek verwahrter Kodex, ${ }^{15}$ liefert mit seinem die Entstehungsgeschichte schildernden Titulus ${ }^{16}$ ein bemerkenswertes Zeugnis für das Aufeinandertreffen von zwei Vorstellungen des Kopierens: einerseits die Vorstellung der Chorherren, die eine Anpassung der Abschrift an den Zeitstil bevorzugten, und andererseits jene des Pfalzgrafen, der sich für die Antike interessierte und das Original so unverstellt wie möglich in seine Abschrift hinüberretten wollte.

Die Darstellung des Gesprächs zwischen Kaiser Hadrian und dem Philosophen Epiktet legt die Unterschiede offen zutage (Abb.1): Während Hadrian auf dem mit Grotesken und Maßwerk überbordenden Thron auf fol. 78 fast verloren wirkt, dominiert die Gestalt des auf einem schlichten Thron unter einer kielbogenartig auslaufenden Arkatur sitzenden Herrschers die Miniatur auf fol. 176v. Dass der Speyerer Buchmaler mit den Putten und Grotesken des Throngestühls die Antike im doppelten Sinne wiederaufleben

Papier, in denen die Instruktionen für die Anfertigung der besagten Miniaturen enthalten waren." ÜS: JCH).

11 Zitiert nach dem grundlegenden Aufsatz zur Notitia dignitatum von Maier 1969, 996; das Zitat ist den Protokollbüchern des Speyerer Domkapitels entnommen: Karlsruhe, Badisches Generallandesarchiv, Prot. 61/10936, 209, cf. http://www.landesarchiv-bw.de/plink/?f=4-756983 (letzter Zugriff 01.10.2017).

12 Maier 1969, 996.

13 Maier 1969, 996.

14 Maier 1969, 997.

15 München, Bayerische Staatsbibliothek, Clm. 10291, cf. Remak-Honnef/Hauke 1991, 154-157. Ein vollständiges Digitalisat der Handschrift ist verfügbar unter: http://daten.digitale-sammlungen.de/ bsb00005863/image_1 (letzter Zugriff 16.04.2015).

16 Der Titulus auf fol. 1 ist bei Maier 1969, $990 \mathrm{f}$. vollständig transkribiert. 


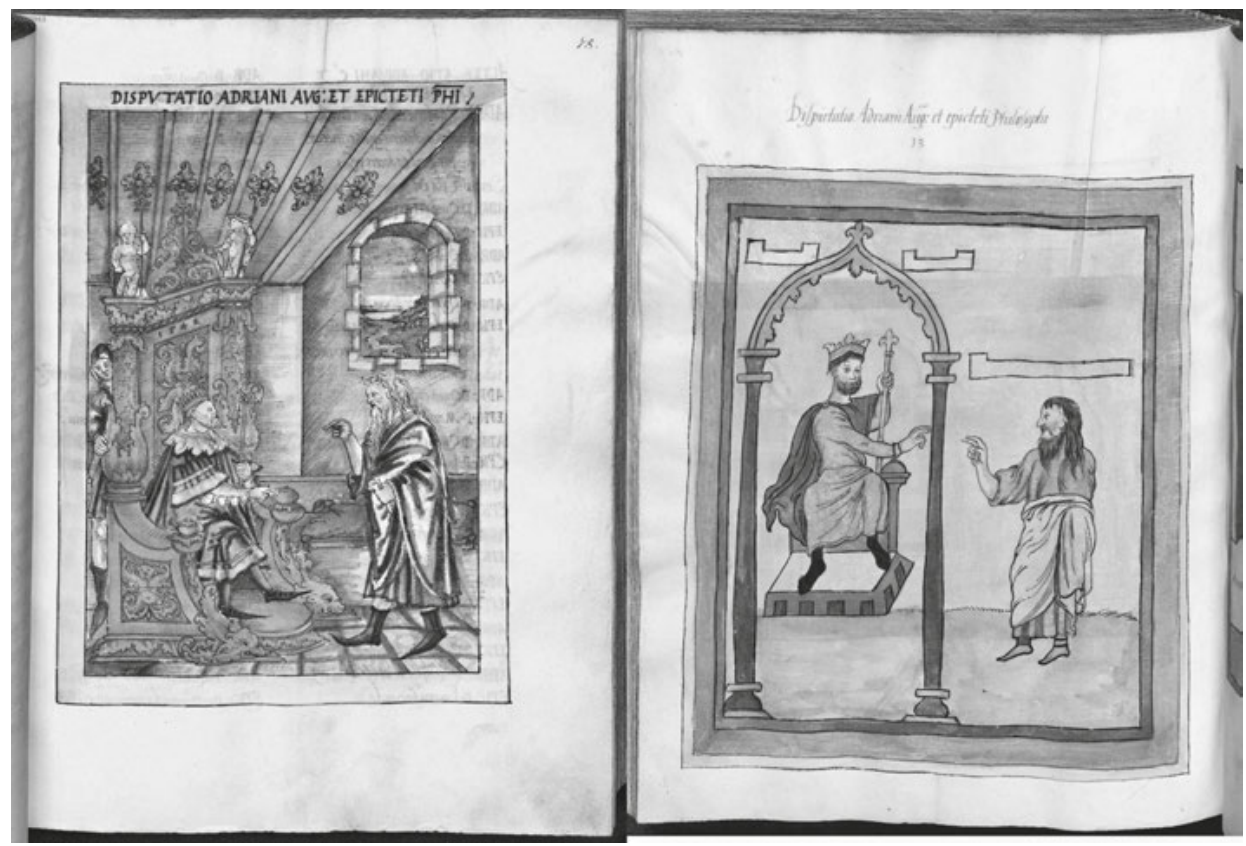

1 Hadrian im Dialog mit dem Philosophen Epiktet; Montage: Notitia dignitatum, München, Bayerische Staatsbibliothek, clm 10291, fol. 78 u. fol. 176v

ließ - im rezipierenden Antikenbezug des deutschen Manierismus ebenso wie im Rückgriff auf die vermeintlich antike Vorlage -, überzeugte den antiquitatis amatorem Ottheinrich offensichtlich nicht. Andererseits ließ sich der antiquarisch interessierte Fürst nicht davon stören, dass die antike Urschrift durch den Zeitstil des ottonischen Kopisten ebenso überlagert gewesen sein mag, wohl auch, weil er den Codex spirensis für ein authentisches Zeugnis antiker Kunstfertigkeit hielt.

Als einziges mir bekanntes Beispiel der spätmittelalterlichen Buchmalerei wird hier der Prozess des liniengenauen Kopierens durch verschiedene Schriftquellen sowie durch die erhaltenen Handschriften selbst dokumentiert. Mehr noch, die Forderung nach einer möglichst exakten Kopie wird von Ottheinrich explizit ausgesprochen. Die unterschiedlichen Wahrnehmungen, was eine Kopie ist, leisten kann und soll, geraten zunehmend in Konflikt zueinander und markieren stellvertretend den epochalen Bruch, der sich asymmetrisch vollzog.

\section{„Unnecessary creativity" oder ein Mangel an Vorlagen?}

Liniengenaue Kopien sind aber keine Neuerung, im Gegenteil, sie kommen in der spätmittelalterlichen Buchmalerei immer wieder vor, nur erfahren wir wenig oder gar nichts 
über sie aus den Schriftzeugnissen, wie das erste Beispiel der von den Brüdern Limburg kopierten Bible moralisée zeigte. ${ }^{17}$ Es ist eine Periode der Kopie vor der im neuzeitlichen Sinne verstandenen Kopie. Gelungene Bildvorlagen werden selbstverständlich tradiert, Texte müssen voneinander kopiert bzw. abgeschrieben werden, um an verschiedenen Orten verfügbar zu sein. Als elementare Technik steht das Kopieren das gesamte Mittelalter durch alle Kunstgattungen hindurch gleichberechtigt neben künstlerischen "Erfindungen", die mitunter sogar auf einen Mangel an Vorlagen zurückgehen mögen.

In den beiden frühesten bekannten und nahezu zeitgleich zwischen 1332 und 1333 gefertigten Exemplaren des Miroir historial, der französischen Übersetzung von Vincent von Beauvais' Speculum historiale, mögen gerade fehlende Vorlagen zu einer voneinander völlig unabhängigen Bildausstattung geführt haben. Die von Claudine ChavannesMazel $^{18}$ erschlossenen Handschriften in Leiden ${ }^{19}$ und Paris ${ }^{20}$ unterscheiden sich bereits hinsichtlich der zu diesem Zeitpunkt gerade erst fertiggestellten Übersetzung des Textes, die im Leidener Kodex auch dank einer aufwendigen Textredaktion dem lateinischen Vorbild nähersteht, während die Pariser Fassung eine vereinfachte, gekürzte Abschrift bereithält.

Die Überraschung besteht darin, dass der Hauptmaler der Leidener Handschrift, der Meister des Jean de Papeleu, nicht nur an der Ausmalung beider Kodizes beteiligt war, sondern in einem Fall denselben Textanfang illustrierte, ohne auf die beim ersten Mal verwendete Komposition zurückzugreifen. ${ }^{21}$ Im Leidener Kodex orientiert sich der Buchmaler auf fol. 104 sehr genau am Text. Dort werden zwei Versionen der Auffindung von Romulus und Remus geschildert, nämlich ihre Entdeckung durch einen Hirten, nachdem sie in einen Fluss geworfen worden waren sowie das viel populärere Narrativ

17 Dass in den Rechnungsbüchern kein Hinweis auf die ältere Handschrift Français 167 enthalten ist, wurde als Argument gegen die Identifizierung von Français 166 mit der in den Quellen erwähnten Bibel angeführt, cf. Dückers 2009, 87. Jonathan Alexander publizierte einen der wenigen überlieferten Verträge, in dem für die Herstellung eines neuen Stundenbuchs explizit die Verwendung einer Vorlage erwähnt wird (Kursivierung durch den Verfasser): „Maistre Jehan Demolin, clerc et escripvain, demeurant à Dijon, doit et promet, par marchié fait, faire à Guillaume le Chamois, bourgeois de Dijon, présent, etc., unes heures de Nostre Dame, contenans en escriptures autant que font celles que lidis Guillaumes lui baille en et pour exemple“ ("Meister Jehan Demolin, Geistlicher und Schreiber, wohnhaft in Dijon, verspricht ein Stundenbuch für Guillaume le Chamois, Bürger der Stadt Dijon, herzustellen, das eine ebensolche Schrift wie jenes besitzt, das besagter Guillaume ihm als Vorbild geliehen hat"), cf. Alexander 1992, 180, ÜS: JCH.

18 Chavannes-Mazal 1988, passim.

19 Leiden, Universitätsbibliothek, Ms. Voss G. G. Fol. 3 A. Ein vollständiges Digitalisat ist verfügbar unter https://socrates.leidenuniv.nl/R/-?func=dbin-jump-full\&object_id=2036625 (letzter Zugriff 21.04.2015).

20 Paris, Bibliothèque nationale, fr. 316. Die Handschrift wurde bislang nicht digitalisiert, ausführliche Angaben zur Literatur finden sich hier: http://www.arlima.net/no/2799 (letzter Zugriff 21.04.2015). Ein vollständiges Digitalisat findet sich hier: http://gallica.bnf.fr/ark:/12148/btv1b10507212h (letzter Zugriff 08.02.2018).

21 Zu den Abbildungen, cf. Chavannes-Mazal 1988, https://www.academia.edu/4964709/The_Miroir_historial_of_Jean_le_Bon._The_Leiden_Manuscript_and_its_Related_Copies_vol_2_PLATES._Dissertation_Leiden (letzter Zugriff 23.04.2015). 
ihrer Auffindung am Bauch der säugenden Wölfin durch den mit seinem Hirtenstab drohenden Faustulus. ${ }^{22}$ Dieselbe Textstelle illustrierte der kennerschaftlich bestimmte Maler in der Pariser Handschrift auf fol. $124 \mathrm{v}$ nur wenige Monate später mit zwei gänzlich neuen Szenen. Er zeigt die Geburt der Zwillinge durch Rhea Silvia sowie den neben der Wölfin stehenden Hirten Faustulus, der die Brüder bereits schützend in seine Arme genommen hat.

Chavannes-Mazel wunderte sich über die "unnecessary creativity", ${ }^{23}$ doch scheinen alle an der Pariser Handschrift beteiligten Maler keinen Zugriff auf das kurz vorher entstandene Leidener Manuskript gehabt zu haben. Ob sie die Illustrationen stattdessen womöglich am Vorbild einer älteren lateinischen Abschrift ausrichteten, sei dahingestellt. Vorlagen scheinen aber gerade bei neu zu illustrierenden Texten nicht unbedingt eine Arbeitserleichterung bedeutet zu haben, denn die Kompositionen waren nur solange im Atelier verfügbar, bis eine Handschrift ihrem Auftraggeber ausgehändigt wurde, es sei denn, der Maler hatte die Kompositionen mittels Kopien für die Werkstatt gesichert. Das würde besonders bei umfangreicheren Aufträgen wie dem Miroir historial mit bis zu knapp 330 Miniaturen etwa in der Pariser Abschrift einen erheblichen Mehraufwand bedeutet haben. Bezeichnend ist daher, dass allein das ebenfalls vom Meister des Jean de Papeleu gemalte Frontispiz im Leidener Kodex überhaupt eine Verbindung zum Pariser Miroir historial herstellt: Bei der Pariser Miniatur handelt es sich um eine getreue Kopie desselben Malers, die allerdings im 15. Jahrhundert wohl aufgrund einer frühen Beschädigung von einem anderen Künstler stark überarbeitet wurde. ${ }^{24}$ Als einzige Miniatur hätte der verantwortliche Maler die Komposition des Frontispizes also mittels eines Vorlagenblattes für andere Gelegenheiten in seinem Atelier aufbewahrt, die übrigen Motive dagegen nicht.

\section{Motivwiederholungen in der Gent-Brügger Buchmalerei}

Bereits seit längerem interessiert sich die Forschung für eine besonders intensive Form der Motivweitergabe in der sogenannten Gent-Brügger Buchmalerei zwischen 1470 und 1550. ${ }^{25}$ Durch die hohe Anzahl an Motivtransfers in dieser Werkgruppe lassen sich anders als in arbiträr versammelten Einzelfällen unterschiedliche Problembereiche getreuer Wiederholungen systematisch eingrenzen und analysieren. Als typisches Beispiel eines vielfachen Motivtransfers wurde das Bordürenmotiv mit der Suche von Maria und Josef nach einer Bleibe in der Weihnachtsnacht (Taf. IV) mehrfach diskutiert. ${ }^{26}$ Das wis-

22 Chavannes-Mazal 1988, 53.

23 Chavannes-Mazal 1988, 144

24 Chavannes-Mazal 1988, 147-176.

25 Die wichtigsten Beiträge zum Problem stammen von: Brinkmann 1988, 90-106; Nash 1998, 12-25; Kren 2005, 357-377.

26 Cf. Lieftinck, 91; London/Los Angeles 2003, cat. no. 21, 147, n. 7; Buck 2006, 103-116; Heyder 2014, 173-179. 
senschaftliche Interesse an diesem Motiv hängt unzweifelhaft mit einer heute in der British Library aufbewahrten und dem Wiener Meister der Maria von Burgund zugeschriebenen Zeichnung zusammen, deren detaillierte Komposition stets als erster Entwurf und Vorlage für die in der Buchmalerei realisierten farbigen Bordüren galt. Erst in jüngerer Zeit sind vor allem wegen der dunklen Grundierung Zweifel an der Eignung des Blattes für den Werkstattgebrauch aufgekommen. ${ }^{27}$ Zwischen 1470 und 1530 entstanden abgesehen von der Londoner Zeichnung mindestens fünfzehn Bordüren nach derselben Grundkomposition. ${ }^{28}$ Davon wurden mindestens acht Bordüren von unterschiedlichen Malern ausgeführt, mehrfach aber griff das Atelier Simon Benings auf das Modell zurück. Mit Ausnahme des ehemals in der Wiener Nationalbibliothek aufbewahrten Rothschild-Stundenbuch ${ }^{29}$ haben die Buchmaler nicht nur die Bordüre, sondern auch die Hauptminiatur illustriert, wenn die Bordüre nicht ohnehin eine Textseite rahmte. Obwohl nicht für alle Exemplare der genaue Entstehungskontext rekonstruiert werden kann, gilt es doch als sicher, dass die Auftraggeber solcher Handschriften in den gesellschaftlich vermögendsten Kreisen zu suchen sind. ${ }^{30}$ Allein ein Exemplar in Brügge ${ }^{31}$ gehört insgesamt einem einfacheren Ausstattungstypus an.

Wie kommt es nun zur Wiederholung eines bestimmten Motivs über einen derart langen Zeitraum und in der hier allein durch die bildliche Gegenüberstellung (Taf. IV) behaupteten Treue zu einem ursprünglichen Entwurf? Was hat die wörtliche Kopie in der Herstellung von Luxushandschriften überhaupt verloren? Widerspricht es nicht dem Prinzip des Extravagant-Herausgehobenen, wenn "nur" etwas nachgeahmt wird, was es bereits gibt oder sitzen wir hier unserem modernen Originalitätsverständnis auf? Was wissen wir überhaupt über das Verhältnis von Käufern und Produzenten mit Blick auf die Anfertigung von Kopien etwa im spätmittelalterlichen Gent und Brügge? Da sich für das Herstellen von Stundenbüchern keine zeitgenössischen schriftlichen Zeugnisse wie im Falle von Ottheinrichs Notitia dignitatum erhalten haben, die das Kopieren einer Miniatur oder gar einer ganzen Handschrift thematisieren, können wir nur aus den Handschriften selber Rückschlüsse auf ihre Produktion und damit auf ein möglicherweise intentionales Kopieren ziehen.

27 Buck 2006, 103-106; Heyder 2014, 173-179.

28 Während Kren in London/Los Angeles 2003, 146 f., Kat.-Nr. 21, acht Varianten zählte, konnte Heyder 2015, 15-20, insgesamt vierzehn Beispiele in der Buchmalerei ausfindig machen. Eine weitere Variante ist dem Verfasser erst jüngst bekanntgeworden. Es handelt sich um eine Wiederholung der Bordüre in einem Stundenbuch von der Hand des Gebetbuchmeisters um 1500 in Brüssel, Bibliothèque Royale, Ms. IV 280, fol. 84v.

29 Ehemals Wien, Österreichische Nationalbibliothek, Cod. ser. n. 2844, inzwischen im Besitz des australischen Geschäftsmannes Kerry Stokes, cf. https://www.nla.gov.au/exhibitions/revealingthe-rothschild-prayer-book (letzter Zugriff 16.04.2015).

30 Die unterschiedlichen Käufergruppen flämischer Luxushandschriften hat Wijsman 2010, 503-550 zusammenfassend dargestellt.

31 Brügge, Openbare Bibliotheek, Hs. 675, fol. 63, cf. http://cabrio.bibliotheek.brugge.be/browse/ webgaleries/MS675/index.html (letzter Zugriff 24.04.2015). 
Die intentionale und treue Wiederholung eines Motives braucht - meines Erachtens immer einen Anlass, einen verständigen Adressaten oder sie verschafft dem Ausführenden einen Vorteil. Bislang ging man davon aus, dass die Gent-Brügger Buchmaler vor allem ihre Arbeitsabläufe ökonomisiert und deswegen immer wieder auf dieselben Vorlagen zurückgegriffen haben. ${ }^{32}$ Diesen Schluss zog man mit Blick auf die niederländische Tafelmalerei der 1450 bis 1480er Jahre, in der sich etwa in der Werkstatt Rogier van der Weydens tatsächlich eine ausgeprägte Kopiertätigkeit entfaltete, die nachweislich auf Grundlage mechanischer Übertragungsmittel, allen voran mit Kartons und Schablonen, geschah. ${ }^{33}$ Doch lassen sich diese Beobachtungen auch auf die Buchmalerei übertragen? Sind die Kopien am Ende nichts weiter als Belege für eine serielle Herstellungsweise ${ }^{34}$

\section{Simon Benings Totenmesse mit Katafalk im Breviarium Grimani}

Für die Simon Bening zugeschriebene Totenmesse mit Katafalk im Breviarium Grimani ${ }^{35}$ (Abb.2) ist kein in allen Details übereinstimmendes Vorbild in älteren Handschriften bekannt. Von einer exakten Kopie im Sinne von Jeltje Dijkstra und Hélène Mund, also einer in zeitlicher wie räumlicher Distanz zum ursprünglichen Entstehungskontext entstandenen Motivwiederholung, kann daher nicht gesprochen werden. ${ }^{36}$ Dafür gibt es mindestens sechs Miniaturen, denen die Fassung im Breviarium Grimani in Bezug auf die Figuren, ihre Gesten oder die perspektivische Darstellung des Katafalks entspricht. ${ }^{37}$ Ein wesentlicher Unterschied zu den älteren Varianten ist allerdings, dass im Breviarium Grimani auf Triforiumshöhe zwar Wappenschilde angebracht sind, jedoch die in den älteren Fassungen an Stangen befestigten Banner mit Wappendarstellungen fehlen.

32 Cf. etwa Nash 1998, 12-25.

33 Ausführliche Darstellungen zu diesem Thema finden sich bei Dijkstra 1990, 70-73; Mund 1992, 66-118 und Kemperdick in: Ausst.-Kat. Berlin/Frankfurt 2008, 95-115.

34 Widersprüchlich sind die Standpunkte hinsichtlich der Untersuchungen zur zeitgleichen Brügger und Antwerpener Tafelmalerei. Während Molly Faries meint, "copying routines and serial production [...]" seien "strong markers for our period [i.e. der untersuchte Zeitraum]", stellt Maximiliaan Martens die immer wieder kolportierte Vorstellung einer seriellen Massenproduktion anhand statistischer Auswertungen massiv in Frage, cf. Faries 2006, 2 sowie Martens 2011, 105-114.

35 Venedig, Biblioteca Marciana, cod. Lat. I, 99 (=2138), fol. 450, cf. Grote 1973, Abb. 58. Jüngst auch: König/Heyder 2016, 139-147.

36 Dijkstra 1990, 32-34; Mund 1992, 122; cf. auch: Mensger 2009/2010, 198-204.

37 a) Zweites Hastings-Stundenbuch, London, British Library, Add. 57492, fol. 184v (Maße: $166 \times$ $121 \mathrm{~mm}$ ); b) Stundenbuch der Isabella von Kastilien, Cleveland, Cleveland Museum of Art, CMA 63256, fol. 219v (Maße: $226 \times 152 \mathrm{~mm}$ ); c) Zweites Stundenbuch des Philipp von Kleve, Wien, Österreichisches Nationalbibliothek, Cod. ser. n. 13239, fol. 152v (Maße: $129 \times 92 \mathrm{~mm}$ ); d) Stundenbuch von Philippe II. Conrault, Oxford, Bodleian Library, Douce 223, fol. 106v (160 × $115 \mathrm{~mm})$; e) Stundenbuch Jakobs IV. von Schottland, Wien, Österreichische Nationalbibliothek, Cod. 1897, fol. 141v (Maße: $200 \times 141 \mathrm{~mm}) ;$ f) La Flora-Stundenbuch, Neapel, Biblioteca Nazionale di Napoli, Ms. I.B.51, fol. 189 (Maße: $204 \times 134 \mathrm{~mm}$ ). 


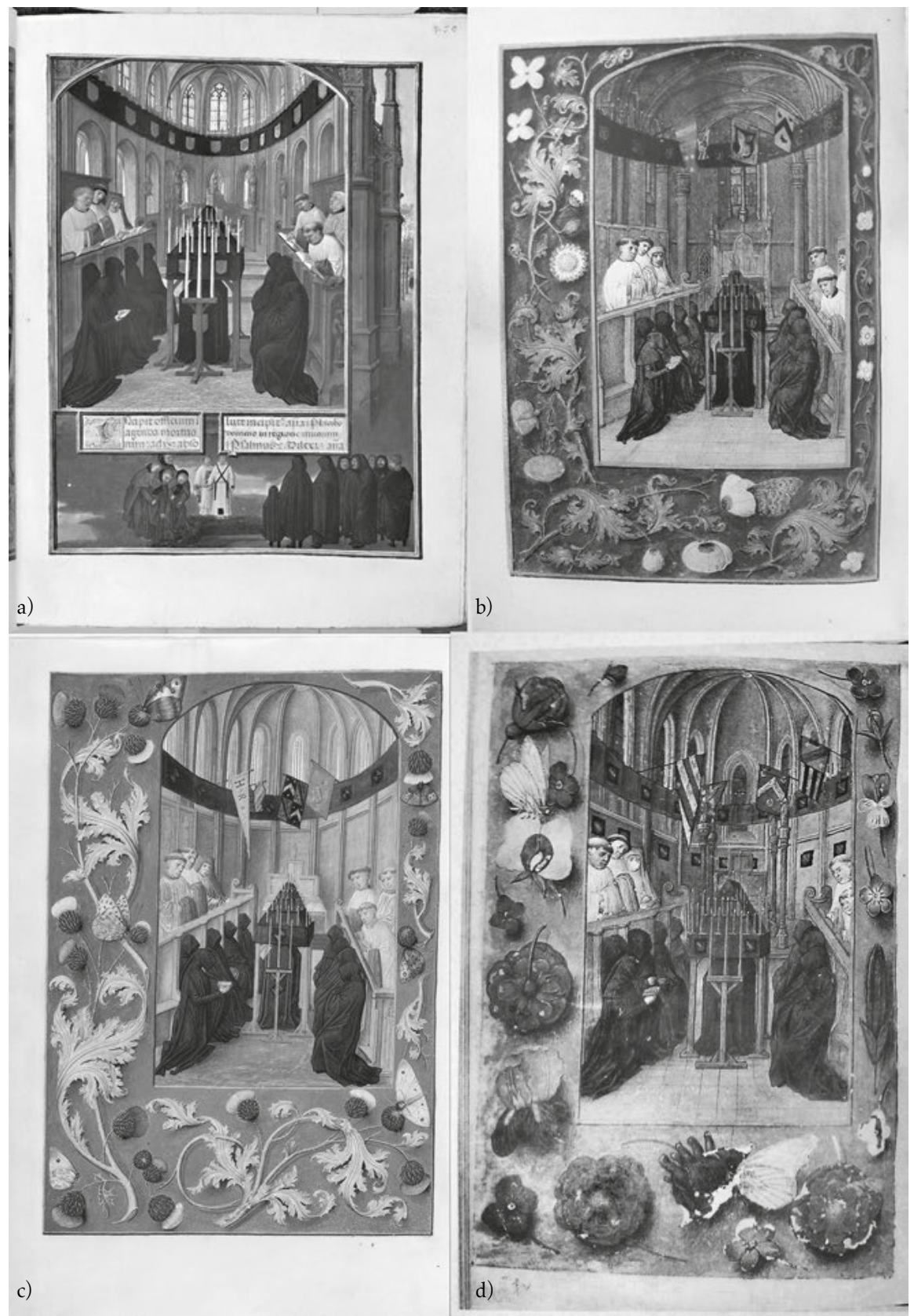

2 Montage (von links oben nach rechts unten): a) Breviarium Grimani, Venedig, cod. Lat. I, 99 (= 2138), fol. 450, Totenmesse mit Katafalk; b) Hastings Stundenbuch, London, Add. 54782, fol. 184v; c) Stundenbuch der Isabella von Kastilien, Cleveland, CMA 63256, fol. 219v; d) Zweites Stundenbuch des Philipp von Kleve, Wien, Österreichische Nationalbibliothek, Cod. 13239, fol. 152v 
Bislang galten von diesen älteren Versionen vor allem die Illustrationen der Totenmesse im Stundenbuch Isabellas von Kastilien in Cleveland ${ }^{38}$ (Abb. 2), im zweiten Hastings-Stundenbuch in London ${ }^{39}$ (Abb.2) sowie im zweiten Stundenbuch Philipps von Kleve in Wien ${ }^{40}$ (Abb.2) als mögliche Anwärter dafür, von Simon Benings Vater Sanders gemalt worden zu sein, der inzwischen sehr entschieden mit dem Meister des älteren Gebetbuchs von Kaiser Maximilian (kurz: Maximilian-Meister) identifiziert wird ${ }^{41}$ Damit kommen sie aufgrund des vermuteten familiären Kontextes als mögliche direkte Vorbilder der Variante im Breviarium Grimani in Frage. Da der Bildausschnitt des Wiener Stundenbuches nicht alle Figuren des ursprünglichen Entwurfs besitzt - statt drei verfügt die Variante nunmehr über zwei Chorherren im rechten Chorgestühl - kann diese Fassung kaum als Vorbild in Frage kommen. Bleiben die beiden Miniaturen in London und Cleveland, von denen die Londoner Fassung spätestens 1483, dem Todesjahr Sir William Hastings, entstanden sein dürfte, während die Miniatur in Cleveland wohl erst in den frühen 1490er Jahren ausgeführt wurde. ${ }^{42}$ Das Londoner Hastings-Stundenbuch besitzt also die früheste bekannte Variante dieser Miniatur, wenngleich diese nicht mit der Urfassung identisch sein muss. So fehlt etwa dem Hastings-Stundenbuch das um den Katafalk verteilte Stroh, das wiederum die Miniaturen im Clevelander Stundenbuch und im Breviarium Grimani enger zusammenrückt. Unverkennbar ist in allen gezeigten Varianten, dass zwar die betenden Chorherren und Pleurants nahezu unverändert in Szene gesetzt werden, das Aussehen des Chors aber permanent variiert: Mal gibt es einen Obergaden, mal gibt es keinen, mal ist die Szene in einen Chor mit Chorumgang platziert, in den meisten Fällen wurde ein polygonaler Chorabschluss bevorzugt. Die Treue hat ihre Grenzen im Detail.

Diese Hauptszene wird im Breviarium Grimani von einer historisierten Bordüre gerahmt, auf der im Bas-de-page (Taf. V) auf der linken Seite ein Grab von Mönchen aus-

38 Cf. hierzu den in Kürze beim Gütersloher Faksimile Verlag erscheinenden Faksimile-Kommentar von Lieve de Kesel sowie De Winter 1981, 342-427. Die Handschrift ist als Volldigitalisat verfügbar unter: http://www.clevelandart.org/art/1963.256 (letzter Zugriff 26.04.2015). Bei De Winter 1981, 347-350, Abb.149-151 findet sich erstmals auch eine Gegenüberstellung verschiedener Fassungen der Totenmesse mit Katafalk.

39 Cf. Turner 1983, passim; Brinkmann 1988, 90-106; Ausst.-Kat. London/Los Angeles 2003, cat. no. 41, 192-194. Brinkmann hat auf Grundlage stilistischer Vergleiche das Londoner Hastings-Stundenbuch aus dem inzwischen zu einer disparaten Gemengelage angewachsenem Euvre des MaximilianMeisters ausgeschlossen und es dem bis dahin nur als Tafelmaler bekannten Meister von 1499 zugeschrieben. Thomas Kren lehnte 2003 diese Zuschreibung ab, wohl auch weil das Londoner Hastings-Stundenbuch eine Vielzahl von Motiven bereithält, die später bei Simon Bening wieder auftauchen. Da keine der überlieferten Varianten der Totenmesse mit Katafalk als "Urfassung" in Frage kommt, ist das sich aus Brinkmanns Zuschreibung ergebende Problem vorerst von nachrangiger Bedeutung.

40 Pächt/Thoss 1990, 90-98; weiterführende Literatur cf. http://data.onb.ac.at/rec/AL00093613 (letzter Zugriff 27.04.2015).

41 Hilger 1973, passim; Van Buren 1975, 286-309; De Winter 1981, 342-427; Ausst.-Kat. London/ Los Angeles 2003, $190 \mathrm{f}$.

42 De Winter 1981, $420 \mathrm{f}$. 
gehoben wird, während zentral platziert ein in Albe und mit gekreuzter Stola gekleideter Priester das Grab mit einem Aspergill einsegnet und rechts Pleurants in Rückenansicht den Verstorbenen beweinen. Dieses Motiv wiederum findet sich wörtlich in einem der frühesten Stundenbücher der Gent-Brügger Buchmalerei des neuen Stils, dem sogenannten Nassau-Stundenbuch in Oxford ${ }^{43}$ auf fol. 214 (Taf. V), das in der Zeit um 1470 gefertigt wurde und heute als das Hauptwerk des Wiener Meisters der Maria von Burgund angesehen wird. Bemerkenswerterweise wurde die auf diesen Entwurf zurückgehende marginale Szene im Breviarium Grimani offenbar vorher nur ein weiteres Mal, nämlich im Brevier Eleonores von Portugal in New York ${ }^{44}$ auf fol. 587, für eine einspaltige Miniatur zur Eröffnung des Totenoffiziums (Taf. V) verwendet. ${ }^{45}$ Diese Miniatur gilt wie das Hastings-Stundenbuch als Werk des Maximilian-Meisters. Jedoch fehlt der Darstellung die im Nassau-Stundenbuch und im Breviarium Grimani so prägnante gekreuzte Stola des Priesters. Zudem ist das in den beiden letztgenannten Fassungen frontal gezeigte Gesicht des Priesters in der New Yorker Variante deutlich nach links gewendet.

Die Kombination von zwei unterschiedlichen Vorlagen für die Ausstattung der Bordüre und des Hauptbildes im Breviarium Grimani setzt einen Maler voraus, dem Vorlagen von unterschiedlichen Künstlern - in diesem Fall vom Maximilian-Meister und vom Wiener Meister der Maria von Burgund - zur Verfügung standen, die er dann neuartig arrangierte. Dabei orientierte sich Simon Bening, der Hauptmaler des Breviariums Grimani, im Detail eng an den ursprünglichen Vorlagen, immer jedoch mit der Freiheit, für eine befriedigende Gesamtkomposition eine eigenständige Lösung zu entwickeln.

Das alles scheint zunächst gut mit dem mittelalterlichen Verständnis des Kopierens vereinbar zu sein, in dem nach Stephan Kemperdick Versatzstücke aus älteren Werken übernommen werden, ohne aber allzu liniengenau zu arbeiten und vor allem ohne die „Kunst", also die besondere stilistische Prägung des älteren Werkes nachzuahmen. ${ }^{46} \mathrm{Da}-$ von abzugrenzen wäre eine exakte Kopie im modernen Sinne, die das ältere Werk sowohl in der Form- und Farbgebung, wie auch in ihrem spezifischen Ausdruck zu imitieren versucht. ${ }^{47}$ Tatsächlich liegt Simon Benings Vorgehensweise aber eher in der Mitte dieser beiden definitorischen Umschreibungen. Das macht sie besonders attraktiv für

43 Nassau-Stundenbuch, Oxford, Bodleian Library, Ms. Douce 219-220, cf. Pächt 1948, 67, Kat.-Nr. 14; Lieftinck 1964, 255-296; Schenk zu Schweinsberg 1975, 139-157; Alexander 1970, o. S.; Ausst.-Kat. London/Los Angeles 2003, 134-137, Kat.-Nr. 18.

44 New York, Pierpont Morgan Library, Ms. Morgan 52, cf. Ausst.-Kat. London/Los Angeles 2003, 321-324, Kat.-Nr. 91.

45 Eine weitere, aber wohl nach dem Breviarium Grimani entstandene Fassung von der Hand des Malers von Den Haag 10 E4 findet sich in den sog. Leber-Hours von Simon Bening (Rouen, Bibliothèque municipale, Ms. 3028 [Leber 142], fol. 179).

46 Kemperdick 2010, $215 \mathrm{f}$.

47 Mensger 2009/2010, 194-221. 


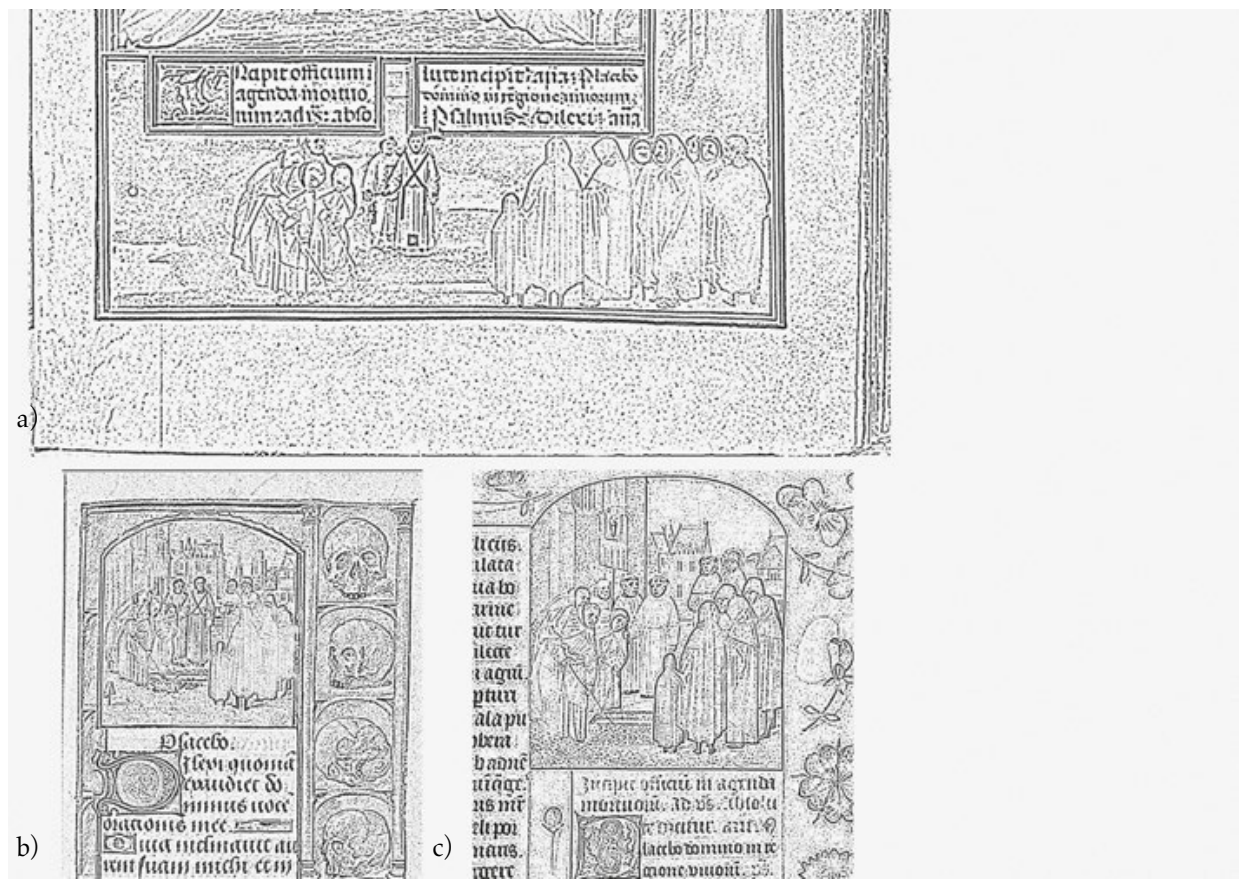

3 Montage (von oben nach rechts unten) der auf die Umrisslinien reduzierten Darstellungen von Taf. V in ihrem originalen Größenverhältnis zueinander

eine experimentelle Analyse des Vorlagengebrauchs, die - so die Intuition - womöglich weitergehende Aufschlüsse über das Wie und Warum vielfacher Motiv-Wiederholungen zu erschließen vermag.

Ein zentrales Kriterium für den Vergleich von vermeintlich liniengenauen Motiven ist ihr tatsächliches Größenverhältnis zueinander. Reduziert man die Miniaturen der eben diskutierten Beerdigungsszene mithilfe eines graphischen Filters zunächst auf die Umrisslinien und stellt sie maßstabsgetreu vergleichend nebeneinander (Abb.3), fallen die proportionalen Abweichungen geringer aus, als es die absoluten Maße der Handschriften zunächst vermuten lassen würden. ${ }^{48}$ Überblendet man in einem weiteren Schritt die charakteristischste Kopie der Komposition im Breviarium Grimani ${ }^{49}$ - den Priester mit gekreuzter Stola - mit der vermuteten Vorlage im Nassau-Stundenbuch

48 a) Breviarium Grimani, Venedig, Biblioteca Marciana, cod. Lat I, 99 (=2138): $280 \times 215 \mathrm{~mm}$; b) Nassau-Stundenbuch, Oxford, Bodleian Library, Ms. Douce 219-220: $138 \times 97 \mathrm{~mm}$; c) Brevier der Eleonore von Portugal, New York, Pierpont Morgan Library, Ms. Morgan 52: $239 \times 173 \mathrm{~mm}$.

49 Die auf ihre Umrisslinien reduzierte Miniatur wurde in der Abbildung interpoliert, damit bei der Überlagerung mit der Miniatur des Nassau-Stundenbuchs die Kontraste sichtbar werden. 

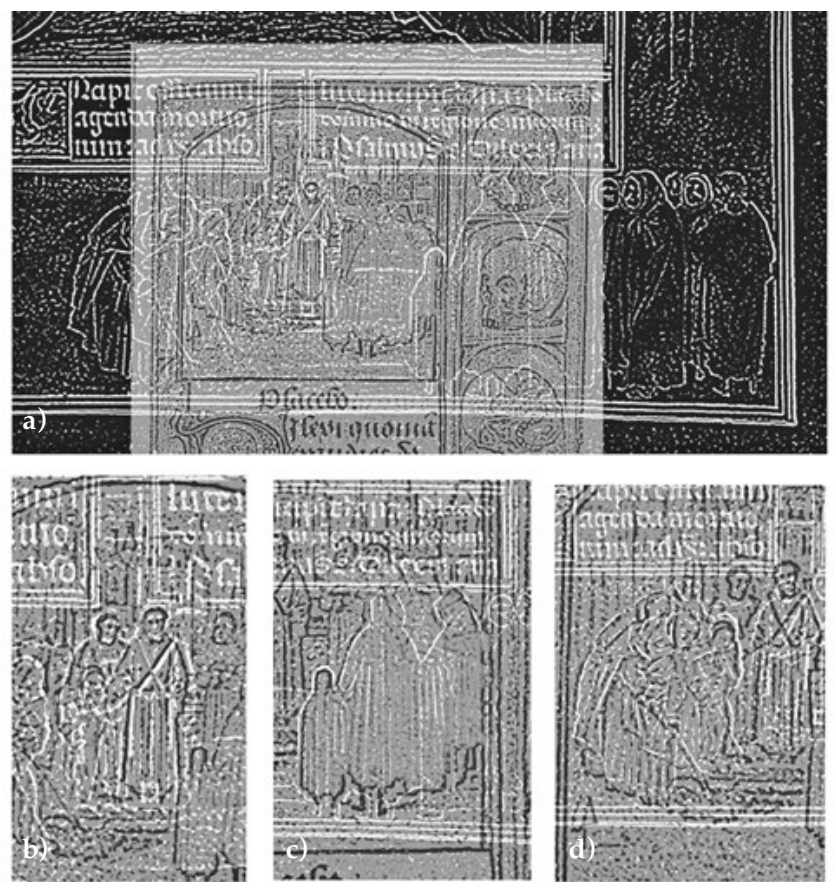

4 Montage (von oben nach rechts unten) von Abb. 3b (schwarze Linien) über Abb. 3a (weiße Linien): a) im originalen Größenverhältnis übereinander geblendet; b) Abb. 3b vergrößert über der Hauptgruppe von $A b b .3 a ; c) A b b .3 b$ vergrößert über die Rückenfiguren von $A b b .3 a ; d) A b b$. 3b vergrößert über die Totengräber von Abb. 3a

(Abb.4a), zeigt sich, dass weder die ältere Miniatur noch eine größengleiche Durchzeichnung hier von Simon Bening verwendet worden sein kann; dafür ist die Miniatur zu klein und die um das Grab gruppierten Pleurants und Totengräber stehen im Nassau-Stundenbuch auch zu eng beieinander. Doch eine proportionale Vergrößerung der Hauptgruppe des Nassau-Stundenbuchs mit Priester und Diakon genügt (Abb.4b), damit sie mit derjenigen im Breviarium Grimani deckungsgleich übereinstimmt. Verschiebt man die Miniatur des Nassau-Stundenbuchs nun von Gruppe zu Gruppe (Abb.4c, d) und vergrößert sie nach Bedarf noch weiter, ergibt sich auch für diese eine liniengenaue Kongruenz. Ähnlich fallen die Beobachtungen für die Miniatur im Brevier der Eleonore von Portugal aus (Abb.5a-c): Legt man das Blatt in Originalgröße auf das Breviarium Grimani, sind die Figuren der New Yorker Miniatur bis auf die Gruppe mit dem Priester zu klein, um als Vorlagen in Frage zu kommen. Vergrößert man sie dagegen, erreicht man wieder - Gruppe für Gruppe - wörtliche Übereinstimmungen.

Welche Schlüsse lassen sich aus diesen Beobachtungen ziehen? Schöpfte Simon Bening in mittelalterlicher Manier aus einem Vorlagenfundus oder entbirgt die getreue, 

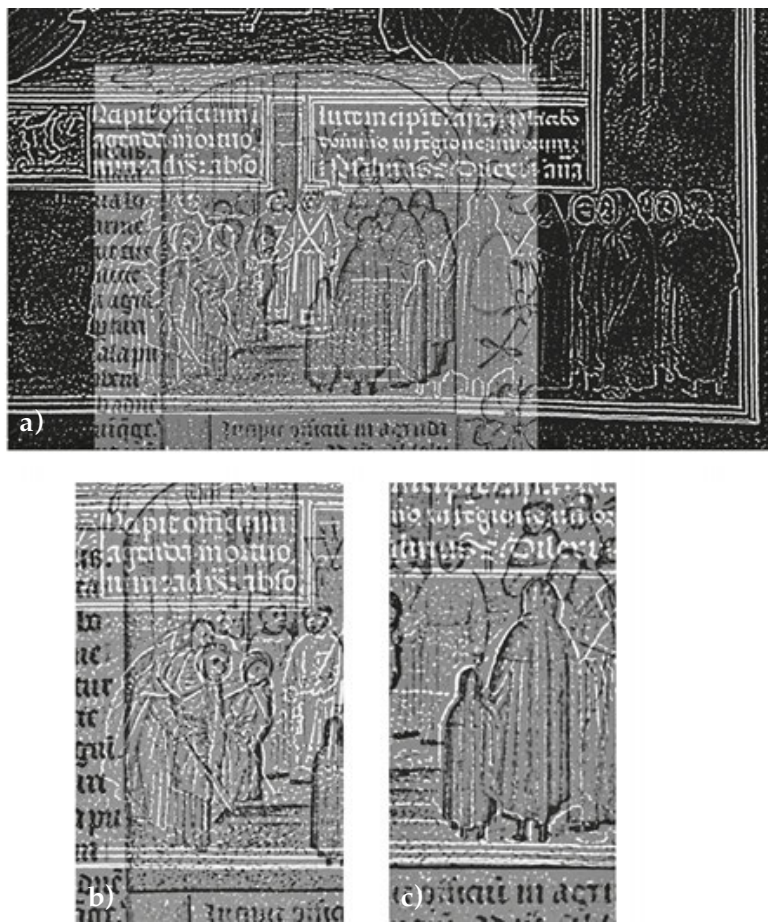

5 Montage (von oben nach rechts unten) von Abb. 3c (schwarze Linien) über Abb. 3a (weiße Linien): a) im originalen Größenverhältnis übereinander geblendet; b) Abb. 3c minimal vergrößert über die Totengräber von Abb. 3a; c) Abb. 3c minimal vergrößert über die Rückenfiguren von Abb. 3a

jedoch größenveränderte und fragmentierte Kopie hier ein gewandeltes Bewusstsein für das Kopieren selbst? Weder wissen wir, wie der Maler die Vorlagen skalierte, noch warum er den älteren Vorlagen soweit folgte, dass er sogar die Faltenstrukturen übernahm. Das ist umso erstaunlicher, da es sich in dem diskutierten Beispiel um eine kleinteilige Illustration einer Bordüre handelt, die in der Ausstattungshierarchie dem Hauptbild nachsteht. Mit der Ökonomisierung der Arbeitsabläufe kann ein solch akribisch und eng an der Vorlage gearbeitetes, aber dennoch individuell angepasstes Bordürenmotiv nicht allzu viel zu tun haben. Nach meiner Kenntnis der im 15. und frühem 16. Jahrhundert bekannten Übertragungstechniken wäre der Gebrauch eines Intermediums vonnöten gewesen, ein Zwischenschritt also, der die Arbeit zwangsläufig verzögert hätte.

\section{Das Problem skalierter Kopien}

Das gilt bereits für die gegen das Licht gepausten Vorlagen, die - bei Cennino Cennini unter dem Begriff carta lucida beschrieben - mittels durchscheinender, geölter Blätter 
aus Pergament oder Papier angefertigt wurden. ${ }^{50}$ Die Technik war offenbar weithin bekannt, wie eine aus der Mitte des 15. Jahrhunderts stammende Beschreibung zur Herstellung von transparentem Pergament in einem Traktat für Schreiber und Buchmaler sowie das eingangs erwähnte Beispiel des Pfalzgrafen Ottheinrich vermuten lassen. ${ }^{51}$ Freilich ließ diese Technik nur größengleiche Übertragungen von Kompositionen zu. In der untersuchten Gruppe scheint dieses naheliegende Hilfsmittel jedoch selten zum Einsatz gekommen zu sein. Zu drastisch fallen die Größenunterschiede zwischen den Handschriften aus: Ein Vergleich aller bekannten Varianten der Totenmesse mit Katafalk fördert die erheblichen Größenunterschiede zwischen den Kodizes zutage, wie besonders deutlich die Gegenüberstellung des größten und kleinsten Kodex der Gruppe zeigt: Das Breviarium Grimani ist mit $280 \times 215 \mathrm{~mm}$ mehr als doppelt so groß wie das zweite Stundenbuch Philipps von Kleve in Wien mit $129 \times 92 \mathrm{~mm} .^{52}$

Von den Techniken, die das Vergrößern oder Verkleinern einer Vorlage zulassen, dürften im späten 15. und frühen 16. Jahrhundert wenn überhaupt Raster verwendet worden sein. Bezeichnenderweise kann diese erstmals bei Masaccio zu beobachtende und spätestens 1538 durch Albrecht Dürers Vnderweysung der messung mit dem zirckel vnd richtscheyt einem größeren Adressatenkreis nördlich der Alpen zugänglich gemachte Übertragungshilfe in keiner einzigen nordalpinen Vorzeichnung des 15. Jahrhunderts nachgewiesen werden. ${ }^{53}$ Andere Techniken des Skalierens, wie etwa der Reduktionskompass oder der Konvexspiegel scheiden wegen der Schwierigkeiten im Umgang mit kleinteiligen Kompositionen aus. Frühe Formen der camera obscura reichen sicherlich in die erste Hälfte des 16. Jahrhunderts zurück, doch auch hier spricht das zumeist kleine Format der Miniaturen und die Vielzahl der zu wiederholenden Kompositionen gegen eine sinnvolle praktische Anwendung.

Keine der uns heute geläufigen Techniken des proportionalen Kopierens scheint zur Hochphase der Gent-Brügger Buchmalerei um 1500 nördlich der Alpen bekannt oder verbreitet gewesen zu sein, sodass eine Verwendung durch die Buchmaler vorausgesetzt

50 Cennini (Milanesi) 1859, 15 f. sowie in dt. Übersetzung bei Cennini (Ilg) 1871, $16 \mathrm{f}$.

51 Graz, Universitätsbibliothek, Ms. 1049, fol. 1v: "Quando igitur vides unam pulchram floraturam in aliquo libro et vis habere eam tecum pro exemplari, pone partem de membrana facta super floraturam et videbis floraturam per membranam et cum stilo exara.i. "reys aus" floraturam sicut eam vides sub memmbrana, et per talem modum potes tecum habere varias floraturas pro exemplari in membranis talibus exaratas." ("Wenn du also in einem Buch ein schönes Ziermotiv siehst und es für dich haben willst als Vorlage, so leg ein Stück der präparierten Haut auf das Motiv, und du wirst das Motiv durch die Haut sehen und kannst es mit dem Griffel nachzeichnen, d. h. ,nachreissen', wie du es unter der Haut siehst, und auf diese Weise kannst du verschiedene Ziermotive als Vorlage bei dir haben, auf solchem Pergament nachgezeichnet." ÜS: Steinmann 2013, 834), cf. Bischoff 1984, 238-240; Steinmann 2013, 834.

52 Cf. die Maßangaben der Handschriften in Fußnote 37 sowie Fußnote 48.

53 Unklarheit herrschte hinsichtlich einer Rogier van der Weyden zugeschriebenen Zeichnung im Museum Boijmans Van Beuningen in Rotterdam, Inv. 9, deren von Sonkes und de Vos als Raster interpretierte Linien in der Grundierung von Fritz Koreny stattdessen auf mögliche Falzungen des Papiers zurückgeführt werden, Ausst.-Kat. Antwerpen 2002, Kat. Nr. 16, 82-84. 
werden könnte. Vielmehr muss zunächst hypothetisch davon ausgegangen werden, dass die meisten Übertragungen tatsächlich freihändig oder unter Zuhilfenahme einer uns heute unbekannten Technik geschahen. Doch auch das freihändige Abzeichnen hätte nicht nur ein ausgesprochen hohes Maß an Geschicklichkeit und Übung erfordert, ${ }^{54}$ sondern vor allem die Bereitschaft, eine Vorlage so genau wie möglich zu wiederholen. ${ }^{55}$ Selbst wenn man den spätmittelalterlichen Buchmalern diese Treue zur Vorlage zutraut, hätte man bei einer freihändigen Übertragung mit proportionalen Verschiebungen oder Verzerrungen zu rechnen, die aber das oben beschriebene Beispiel in den Einzelgruppen gerade nicht aufweist (Abb. $4 \mathrm{a}-\mathrm{d}$ ).

Es könnte sich um einen Zufall handeln, um eine rare Ausnahme. Allerdings ist die liniengenaue proportionale Vergrößerung bzw. Verkleinerung auch in vielen anderen Beispielen nachzuweisen, wie ein Vergleich der Hauptbilder mit der Darstellung der Totenmesse mit Katafalk bezeugt. Bemerkenswert ist dabei, dass die Handschriften mit den entsprechenden Varianten bezogen auf ihre Provenienz und Zeitstellung oft keine direkten Verbindungen aufweisen. Dafür lässt sich eine mal lose, mal engere Verbindung der Maler zueinander in den meisten Fällen rekonstruieren. Die Form der Übernahme scheint dabei nicht immer nach demselben Muster zu verlaufen. Im Stundenbuch der Isabella von Kastilien in Cleveland übertrug der Maler nur einzelne Figurengruppe der Komposition, die ihm aus dem Londoner Hastings-Stundenbuch oder einem Vorlagenblatt bekannt waren (Abb. 6a-d). Die Miniatur im Oxforder Stundenbuch des Philipp II. Conrault ${ }^{56}$ wiederum erweist sich als minimal vergrößerte, dafür aber hinsichtlich ihrer räumlichen Disposition in den Grundzügen übertragene Komposition mit verkleinertem Bildausschnitt des im Hastings-Stundenbuch bewahrten Entwurfs der Szene (Abb. 7a-c). ${ }^{57}$ Eine Übertragung konnte demnach von einzelnen Figuren, Gruppen, (Stadt-)Landschaften bis hin zu ganzen Kompositionen reichen.

\section{Methodischer Einwand}

Untersuchungen von Motivwiederholungen betreiben zwangsläufig eine mediale Illusion den zeitgenössischen Betrachtern war das Nebeneinander der vielfachen Wiederholungen nicht in gleicher Weise möglich, wie den heutigen Betrachtern. Das uns so selbstverständliche Vergleichen dürfte zur Entstehungszeit der Werke Ausnahmefall geblieben sein; es ist noch am ehesten im unmittelbaren Entstehungskontext der Werkstatt denkbar. Das

54 Berühmt ist der Ausspruch Albrecht Dürers: „Item muß er [der Lehrknabe] von guter Werkleut Kunst erstlich viel abmachen, bis dass er eine freie Hand erlangt", cf. Meder 1923, 256.

55 Ausst.-Kat. München/Heidelberg 2014, 111-116.

56 Oxford, Bodleian Library, Ms. Douce 223, cf. Ausst.-Kat. London/Los Angeles 2003, $188 \mathrm{f}$.

57 Dem ungewöhnlich in die Länge gezogenen Chor, der charakteristisch für die Raumauffassung im gesamten Londoner Hastings-Stundenbuch steht, wollte oder konnte der Maler des Oxforder Stundenbuchs allerdings nicht folgen. Als Vorlage kommt allein deshalb schon ein Intermedium in Frage. 


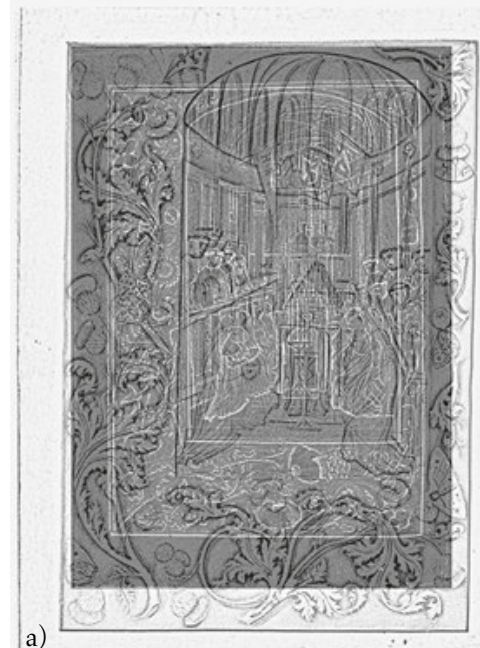

a)
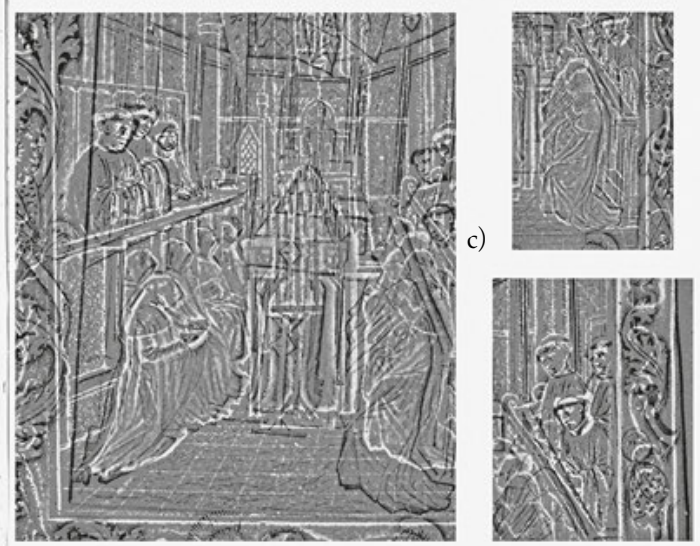

b)

d)

6 Montage (von links nach rechts unten) von Abb. 2c (schwarze Linien) über Abb. 2b (weiße Linien): a) im originalen Größenverhältnis übereinander geblendet; b) Abb. 2c verkleinert über den Chorherren und Pleurants auf der linken Seite von Abb. 2b; c) Abb. 3c verkleinert über den Pleurants auf der linken Seite von Abb. 2b; d) Abb. 2c verkleinert über den Chorherren auf der linken Seite von Abb. 2b

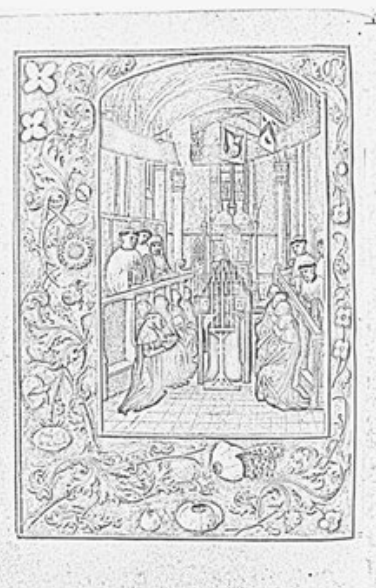

a)

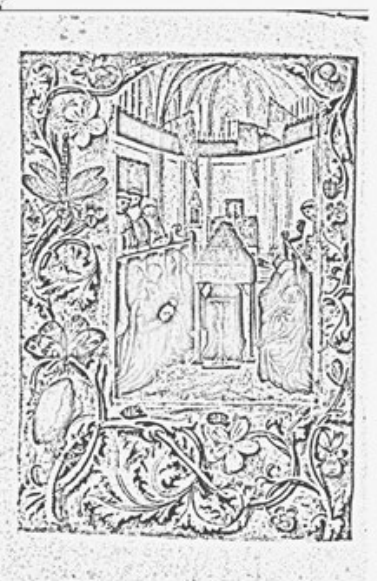

b)

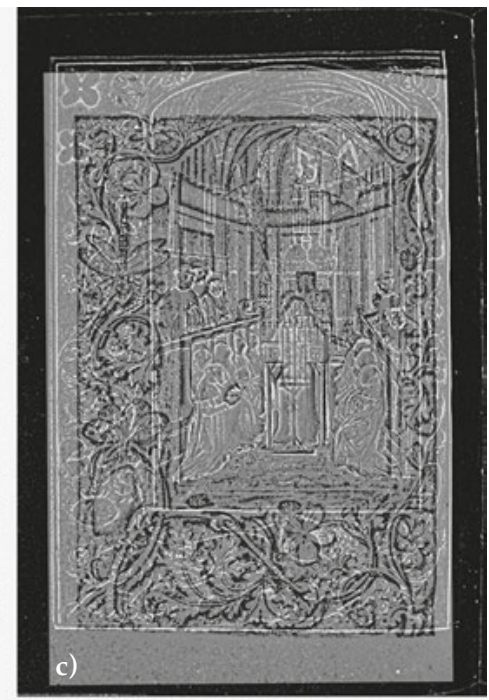

7 Montage (von links nach rechts) der auf die Umrisslinien reduzierten und in ihren originalen Größenverhältnissen dargestellten Miniaturen: a) Abb. 2b; b) Stundenbuch von Philippe II. Conrault, Oxford, Bodleian Library, Douce 223, fol. 106v; c) Abb. 7b (schwarze Linien) minimal verkleinert über Abb. 2b (weiße Linien) 
Movens für eine Motivkopie wäre zunächst nicht ursächlich in der Ähnlichkeit zu ihrem Vorbild zu suchen. Das Nebeneinander vieler gleichartiger Versionen konnte erst den Connoisseur in der historischen Betrachtung überraschen. Gleichwohl büßt die vielfache linientreue Wiederholung vor dem Hintergrund unzähliger nur in Nuancen anders gestalteter Kompositionen der Totenmesse mit Katafalk ${ }^{58}$ ihre Attraktion trotz dieses Einwandes nicht ein. Ganz im Gegenteil: Die hohe Verbindlichkeit zur Vorlage, die lineare Präzision verlangt nach einem Grund, warum für ihre Realisierung weder Zeit noch Mühe gespart wurden. Konnten einzelne Kompositionen innerhalb der Buchmalerei im frühen 16. Jahrhundert etwa genauso wie ein Altarbild in den Ruf geraten, die Erfindung eines berühmten Künstlers zu sein, sodass ein Auftraggeber oder Agent sich - wie etwa für Rogiers Madrider Kreuzabnahme belegt ${ }^{59}$ - explizit um eine Kopie des Werkes bemühte?

Vergleichbare Fälle mag es gegeben haben: Den berühmten Kalender im Breviarium Grimani hat der Buchmaler Gerard Horenbout zweifellos nach dem Vorbild der Très Riches Heures des Herzogs von Berry gestaltet. ${ }^{60}$ Diese befanden sich in der Entstehungszeit des Breviers in den 1510er Jahren aller Wahrscheinlichkeit nach in der Sammlung Margarete von Österreichs, die, wenn nicht selber Auftraggeberin des Breviers, zumindest den Zugang zum Kodex gewährt haben muss. Besonders der Abstand zur Entstehungszeit der Très Riches Heures liefert ein belastbares Indiz für die ungebrochene Wertschätzung der Malerei der Brüder Limburg. Anders als im Fall der Kopien der großen Kreuzabnahme Rogiers ahmte Gerard Horenbout aber nicht die Kunst der über hundertjährigen Vorlage nach, sondern aktualisierte die Kompositionen und übertrug sie in den eigenen Zeitgeschmack; Liniengenauigkeit spielte für ihn dabei keine Rolle. Damit unterscheidet sich seine Annäherung an die Vorlage entscheidend von derjenigen Simon Benings, der zumindest partiell die zum Vorbild genommene Kunst nicht nur liniengetreu, sondern auch in ihren farblichen und räumlichen Charakteristika nachahmte.

58 Eigenständige Lösungen der Totenmesse mit Katafalk finden sich beispielsweise in: a) Madrid, Biblioteca Nacional de España, Vitr. 24-2, fol. 139v; b) Berlin, SBB-PK, Ms. theol. lat. fol. 285, fol. 587v; c) Cambridge/Mass., Houghton Library, Richardson Ms. 9, fol. 140v; Antiquariat Tenschert, Leuchtendes Mittelalter III, Nr. 23, fol. 74.

59 Cf. Kemperdick 2010, 207-230; Mensger 2009/2010, 194-221. Antonia Putzger unterzieht in einem zentralen Kapitel ihrer vor der Veröffentlichung stehenden Dissertation die verworrene (historische) Gemengelage von erhaltenen und in den Quellen bezeugten Kopien der großen Kreuzabnahme Rogiers einer kritischen Revision.

60 Durrieu 1903, 321-328; dazu abweichend Krieger 2012, 383-485 (bes. 438 f., 485), der zufolge der Kalender im Breviarium Grimani sowie alle weiteren, dem Meister Jakobs IV./Gerard Horenbout zugeschriebenen Miniaturen desselben Breviers von einem eigenständigen Maler ausgeführt wurden. Die Autorin klammert bei ihren Überlegungen die historischen Umstände für das Kopieren des Kalenders der Brüder Limburg allerdings völlig aus. Jüngst dazu: König/Heyder 2016, 71-103. 


\section{Exkurs und Ausblick: die Kopie als Erfahrung von Differenz}

Die Suche nach guten Gründen für die liniengenaue, größenveränderte Wiederholung in der Gent-Brügger Buchmalerei kann ihren spekulativen Charakter kaum verhehlen. Jedoch erscheinen diese Wiederholungen insbesondere im Kontrast zu den viel freier umgesetzten Nachahmungen Gerard Horenbouts umso rätselhafter. Ein möglicher Schlüssel zum Verständnis des Mehraufwands mag in der wesenhaften Abhängigkeit der Kopie von ihrer Rezeption liegen. ${ }^{61}$ Für eine Reflexion der Wahrnehmung und Beurteilung von Wiederholungen (i.e. ihre Rezeption) bieten ausgerechnet die einesteils zu "Popikonen“ erhobenen, anderenteils kritisch beäugten Philosophen der Differenz die vielleicht vielversprechendsten Ideen. Der Exkurs zu einem zentralen Denkansatz der Philosophie des 20. Jahrhunderts mag daher vor allem als katalysatorisches Instrument verstanden werden.

Im Jahr 1968 veröffentlichte Gilles Deleuze seine Abhandlung Differenz und Wiederholung $^{62}$ über das Wesen und die Bedingungen der "Differenz", die das seit Platon in der westlichen Philosophie gültige Primat von Identität und Repräsentation infrage stellte. Nicht als dialektisches Gegenstück, sondern als zentrale Komponente des Differenzbegriffes diskutierte der Autor dabei die Bedeutung der "Wiederholung” in kritischer Lektüre des Begriffs bei Immanuel Kant, Sören Kierkegaard, Friedrich Nietzsche und Henri Bergson. Der Anspruch der Studie zielt dabei unmittelbar auf das "Sein", das sich nach Deleuze „von der Differenz selbst ${ }^{\text {“63 }}$ aussagt. Der dichte und an vielen Stellen sicher auch dunkle und mitunter gar zum Widerspruch neigende Text ist kaum auf zentrale Aussagen zu reduzieren und selbst sympathisierende Denker wie Michel Foucault bemühten sich nicht um eine konsistente Wiedergabe des Inhalts. ${ }^{64}$ Daher möchte ich mich auf einige wenige, notgedrungen aus dem Kontext gerissene Überlegungen konzentrieren, die die bisherigen Beobachtungen vor allem hinsichtlich der Rezeption von Kopien bereichern mögen.

Deleuze bricht grundsätzlich mit der Vorstellung des Originals, da es immer schon Bezugspunkte gebe, auf die auch ein Original rekurriert. Der Autor wendet sich gegen die platonische Bewertung von Bildern zugunsten einer fundamentalen Aufwertung des Trugbildes ("simulacrum"), das in seiner Lesart "den wahren Charakter oder die Form

61 Ein vergleichbares Gewicht auf das rezeptive Moment von Kopien legte Walter Cupperi in seiner vielschichtigen Einleitung, cf. Cupperi 2014, 13.

62 Originaltitel: Différence et répétition: Deleuze 1968 und in deutscher Übersetzung durch Jürgen Vogl: Deleuze 1992.

63 Deleuze 1992, 59. Im französischsprachigen Original: „L'Être se dit en un seul et même sens de tout ce dont il se dit, mais ce dont il se dit diffère: il se dit de la différence elle-même.", cf. Deleuze 1968, 53.

64 Foucault 1977, 7-12. Weiterführende Literatur zum Thema: Kimmerle 2000, passim [83-115], Münker/Roesler 2000, 49-58; Heyer 2001, passim; McMahon 2005, 42-52; Otto 2007, 257-324; Parr (Hg.) 2013, 15-17, 74 f., 181 f., 225 f., 254-256, 276-278. 
dessen, was ist - des ,Seienden' -“, 65 repräsentiert: „Umkehrung des Platonismus meint hier: das Primat eines Originals gegenüber dem Abbild, eines Urbilds gegenüber dem Bild anfechten. Das Reich der Trugbilder und Spiegelungen verherrlichen. "66 Die schroffe Ablehnung der Ausrichtung auf ein Ursprüngliches, zugunsten eines an Friedrich Nietzsche orientierten Modells der ewigen Wiederkunft, mag in einer historischen Wissenschaft wie der Kunstgeschichte zunächst verstörend und irreführend wirken. Doch auf der anderen Seite resultiert aus ihr für jedes Artefakt - und eben auch für alle Kopien eine unvoreingenommene Betrachtung. Hinzu kommt, dass sich das Wiederholen nach Deleuze immer "zu etwas Einzigartigem oder Singulärem, das mit nichts anderem ähnlich oder äquivalent ist", ${ }^{67}$ verhält und für das kein "zuschreibbare[r] Ursprung"68 ausgemacht werden kann. Diese Bestimmung des Begriffes scheint der üblichen Lesart von Wiederholungen zu widersprechen, wird sie doch normalerweise genau über ihre Referentialität zu einem zeitlich und materiell Vorgängigen definiert. Entsprechend räumt Deleuze ein: „Die Wiederholung läßt sich stets als eine äußerste Ähnlichkeit oder eine vollendete Äquivalenz, repräsentieren'. Aber die Tatsache, daß man in winzigen Schritten von einer Sache zur anderen gelangt, verschlägt nicht, daß eine Wesensdifferenz zwischen beiden besteht. ${ }^{169}$ Der Kerngedanke lautet folglich, dass ein Wiederholtes stets ein Neues, ein Differentes sein muss, da es sonst mit dem zu Wiederholenden zusammenfiele, mit ihm identisch sei. Mehr also als die Ähnlichkeit oder Analogie würde die Wiederholung (und damit auch die Kopie) erst durch ihre Differenz aufscheinen.

Diese Überlegung lässt sich auf die Rezeption von Kunst im Allgemeinen und die spätmittelalterliche Buchmalerei im Speziellen übertragen und tatsächlich werden alle vorgestellten Beispiele erst in der Erfahrung ihrer Differenz wahrgenommen. Das heißt: Wir werden eine Wiederholung überhaupt erst als solche bezeichnen, wenn uns Unterschiede trotz „äußerste[r] Ähnlichkeit[en]“ auffallen. Es gehört zur medialen Bedingung von Kopien, sich auf ein Anderes stets zu beziehen und sich zugleich von diesem Anderen zu unterscheiden. Deleuze betrachtete - mit Verweis auf die Philosophie Gabriel Tardes - deshalb "die Wiederholung als das Differenzierende der Differenz". ${ }^{70}$

Das setzt aber für unsere Überlegungen voraus, dass es diese Referenzwerke für den Betrachter überhaupt gegeben hat. Kopien wurden entweder als solche wahrgenommen, oder sie sind gerade nicht wahrgenommen worden, weil sie in Unkenntnis der Zweioder $\mathrm{n}$-fachheit verkauft und bewundert wurden. Eine dritte Lösung wäre denkbar: Die Wiederholung bzw. Kopie wurde vielleicht nicht unmittelbar, aber doch in einem allge-

65 Deleuze 1992, 95.

66 Deleuze 1992, 95. Zur Auseinandersetzung Deleuzes mit dem Platonismus, cf. vor allem Otto 2007, 269-278.

67 Deleuze 1992, 15.

68 Deleuze 1992, 164.

69 Deleuze 1992, 16.

70 Deleuze 1992, $106 \mathrm{f}$. Tarde propagiert das Zusammenspiel zwischen Wiederholung und Differenz als ein universelles Prinzip: Tarde 1890, Kap. I "La Répétition universelle". 
meinen Verständnis als Träger einer älteren Bildidee verstanden. Hier könnte eine Lesart verborgen liegen, die sich auch in der niederländischen Tafelmalerei beobachten lässt: Der Jahrzehnte währende Gebrauch alter Vorlagen durch Maler wie Simon Bening, die durchaus in der Lage waren, eigenständige Bildideen zu entwickeln, beruft sich mit seiner hohen Verbindlichkeit gegenüber alten Vorlagen in einer Periode des gesellschaftlichen und religiösen Umbruchs auf die "gute alte Zeit". Um die Mitte des 16. Jahrhunderts greift in der Kunstproduktion massiv ein flämischer Archaismus ${ }^{71}$ um sich, für den Künstler wie Marcellus Coffermanns ${ }^{72}$ oder Verleger wie Hieronymus Cock ${ }^{73}$ stellvertretend genannt seien und den erstmals Erwin Panofsky beschrieben hat. ${ }^{74}$ In der Gent-Brügger Buchmalerei sind Vorboten dieses Archaismus bereits um 1500 nachweisbar; sie prägen die Kunstform, die im Umfeld des erblühenden Buchdrucks selbst bald nur noch als kostspieliger Archaismus wahrgenommen wurde.

Hierzu passt, dass Simon Bening im Beatty-Rosarium (Abb. 8a) ${ }^{75}$ die Madonna in der Kirche von Jan van Eyck ${ }^{76}$ (Abb. 8b) zumindest teilweise wiederholte. Zur Entstehungszeit der Miniatur in den 1530er Jahren zählte das vermeintliche Vorbild also bereits knapp hundert Jahre. Erstaunlicherweise ging Bening, selbst ein ausgezeichneter Lichtmaler, gerade auf die wichtigste Errungenschaft des Bildes nicht ein: das "Inslichtsetzen" des Kirchenraums. Die ganzfigurige Darstellung der Vorlage wandelte der Maler in einem Close-up zu einem halbfigurigen intimen Porträt der gekrönten Gottesmutter mit einem andersfarbigen Gewand ab und setzte es vor einen hellblauen, nicht näher zu bestimmenden Bildgrund. Zumindest das Gesicht lässt den Eindruck entstehen, hier habe der Maler den künstlerischen Modus des älteren Bildes nachahmen wollen.

Die vielen Abweichungen nähren die Skepsis, ob in Jan van Eycks Bild tatsächlich die Vorlage zu suchen ist, entstanden doch am Beginn des 16. Jahrhunderts bereits andere Nachahmungen dieser offenkundig noch immer berühmten Tafel. So ließ sich etwa der italienische Gesandte und Kunstagent Antonio Siciliano eine Kopie der Komposition von Jan Gossart beziehungsweise Gerard David anfertigen (Abb. 8c). ${ }^{77}$ Es darf in diesem Zusammenhang nicht unerwähnt bleiben, dass besagter Antonio Siciliano auch mit der Entstehungsgeschichte des Breviariums Grimani verbunden ist. Für den enormen Preis von 500 Golddukaten hatte er den prachtvollen Kodex an Kardinal Domenico Grimani verkauft - jedoch nicht, ohne vorher gewitzt sein eigenes Wappen in eine der unzähli-

71 Cf. z. B. Van den Brink 2001, 12-43; Mensger 2001, 44-51; Powell 2006, 707-728.

72 Vrij 2003, passim.

73 Cf. z. B. Van Grieken 2008, 29-45.

74 Panofsky 1953/2001, 354-360. Frühe Bestrebungen zu einer Orientierung an den Gründervätern der flämischen Malerei datiert Panofsky in die Jahre um 1500.

75 Dublin, Chester Beatty Library, Ms. Western 99, cf. Testa 1984, 189-136.

76 Berlin, SMPK, Gemäldegalerie, Inv. 525C, zw. 1437-1439, Öl auf Eichenholz, $31 \times 14 \mathrm{~cm}$.

77 Rom, Galleria Dora Pamphilj, Öl auf Eichenholz, beide Tafeln: 40,2 × 22 cm, zw. 1510-1515, cf. Ausst.-Kat. New York/London 2010, 140-145, Kat.-Nr. 7a-b. 

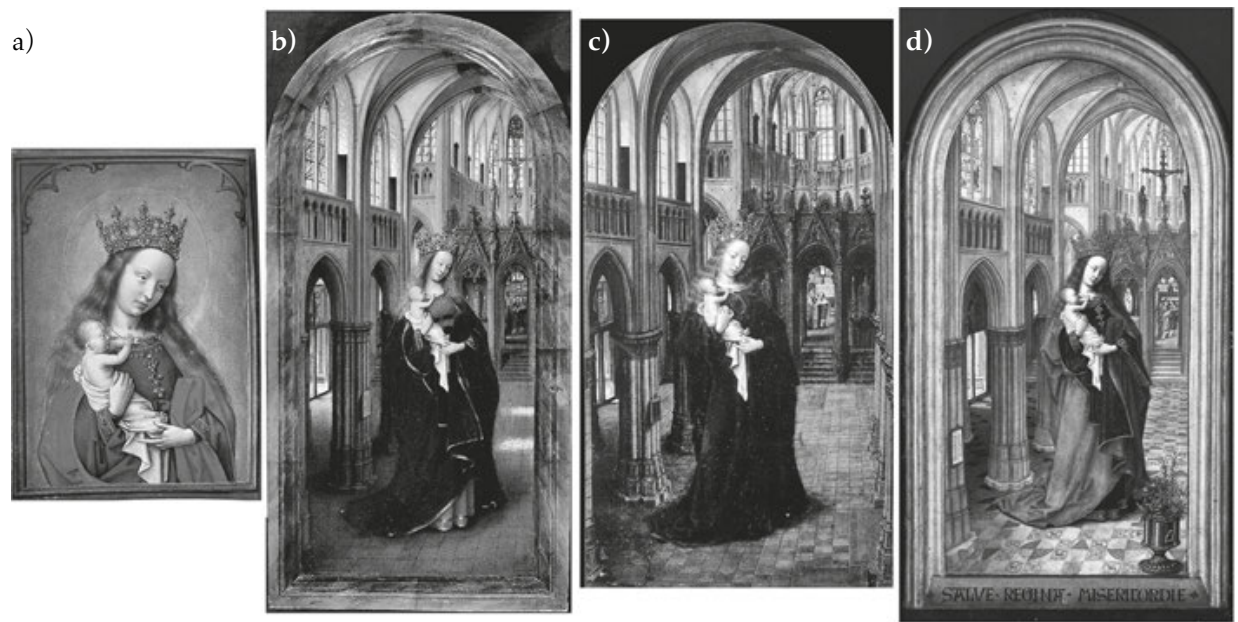

8 Montage (von links nach rechts): a) Beatty-Rosarium, Dublin, Chester Beatty Library, Ms. Western 99, fol. 44v; b) Berlin, SMPK, Gemäldegalerie, Inv. Nr. 525C, Öl auf Eichenholz, $31 \times 14 \mathrm{~cm}$, zw. 1437-1439; c) Rom, Galleria Dora Pamphilj, Öl auf Eichenholz, beide Tafeln: 40,2 × 22 cm, zw. 1510-1515; d) Antwerpen, Koninklijk Museum voor Schone Kunsten, Inv./Kat.-Nr. 255-256, Öl auf Holz, 1499

gen Textbordüren unterbringen zu lassen. ${ }^{78} \mathrm{Da}$ er also spätestens in der Endphase der Herstellung mit dem ausführenden Atelier in Kontakt gestanden haben muss, kannte er ohne Zweifel auch den Hauptmaler der Handschrift: Simon Bening. Der gemeinsame Käufer bzw. Auftraggeber rückt die drei Maler (David, Gossart, Bening) daher noch enger zusammen, als sie aufgrund stilistischer Überschneidungen und Kompositionsübernahmen ohnehin in der Vergangenheit diskutiert wurden. Der Vergleich des Tafelbildes mit der Miniatur macht aber deutlich: Die Mantelfarbe folgt im Diptychon für Antonio Siciliano dem eyckischen Original.

Das gilt nicht für das 1499 für den Abt der Duinenabtei, Christian de Hondt, entstandene Diptychon vom eponymen Meister von 1499 (Abb. 8d). ${ }^{79}$ Hier trägt Maria sowohl den in der Miniatur so herausstechend leuchtend roten Mantel als auch ein blaues Kleid mit grünem Innenfutter. Greifbar wird ein "Stille-Post-Spiel“, das im Falle von Benings "Kopie“ den vermeintlichen Ursprung, eben das Werk des Meisters von 1499, in künstle-

78 Das Wappen ist in die Bordüre einer Textseite des Temporales auf fol. 81 eingemalt. Abbildungen bei Coggiola 1908, 61 sowie König/Heyder 2016, 15, Abb. 4.

79 Antwerpen, Koninklijk Museum voor Schone Kunsten, Inv./Kat.-Nr. 255 f., Öl auf Holz, 1499, cf. Eeckhout 1985/1988, 49-62. 
rischer Qualität zu überflügeln vermag. ${ }^{80}$ An diesem Beispiel wird die Überlegung zur Differenzerfahrung augenfällig. Der Besteller mag das Dipytchon des Christian de Hondt gekannt haben und womöglich sogar - zumindest vom Hörensagen - das eyckische Original: Bening passte das Bild in die für ihn typische Miniaturrahmung ein und bemühte sich darüber hinaus auch um eine stilistische Nähe zum Vorbild, zumindest aber um Liniengenauigkeit. Wäre es denkbar, dass der Besteller gerade die Kombination aus der Nähe zum Vorbild mit dem Differenten, dem typischen „Bening-Schmelz" besonders zu schätzen wusste? Das würde, als Verkaufsargument zumindest, den Vorgang der liniengenauen Kopie ansatzweise erklären: Das Erzeugen eines wiedererkennbaren Produkts für das Auge des kenntnisreichen, auf Repräsentation bedachten Auftraggebers. Es würde aber auch aufzeigen, dass es hier ein offensichtliches sich zu einer Kopie verhalten gibt und allein dieser Aspekt leitet - wenn auch unter anderen Vorzeichen - ebenso in die Neuzeit, wie die eingangs vorgestellten Kopien "uf die alt handt” des Pfalzgrafen Ottheinrich.

\section{Bibliografie}

Alexander, Jonathan J. G.: The master of Mary Burgundy. A book of hours for Engelbert of Nassau, The Bodleian Library, Oxford, New York 1970.

Alexander, Jonathan J. G.: Medieval illuminators and their methods of work, New Haven [u.a.] 1992.

Ausst.-Kat. Early Netherlandish drawings from Jan van Eyck to Hieronymus Bosch (Antwerpen, Rubenshuis 2002), hg. v. Fritz Koreny, Antwerpen 2002.

Ausst.-Kat. Illuminating the Renaissance. The triumph of Flemish manuscript painting in Europe (Los Angeles, J. Paul Getty Museum 2003; London, Royal Academy of Arts (2003/2004), hg. v. Thomas Kren, Scot McKendrick und Maryan Wynn Ainsworth, Los Angeles 2003.

Ausst.-Kat. Der Meister von Flémalle und Rogier van der Weyden (Frankfurt a. M., Städel Museum 2008/2009; Berlin, Gemäldegalerie 2009), hg. v. Stephan Kemperdick und Jochen Sander, Ostfildern 2008.

Ausst.-Kat. Man, myth, and sensual pleasures. Jan Gossart's Renaissance. The Complete Works (New York, Metropolitan Museum of Art 2010/2011; London, National Gallery 2011), hg. v. Maryan W. Ainsworth, New York [u. a.] 2010.

Ausst.-Kat. Punkt, Punkt, Komma, Strich. Zeichenbücher in Europa, ca. 1525-1925 (München, Zentralinstitut für Kunstgeschichte 2014; Heidelberg, Universitätsbibliothek 2015/2016), hg. v. Maria Heilmann [u.a.], Passau 2014, URL: http://archiv.ub.uni-heidelberg.de/artdok/volltexte/ 2015/3344 (letzter Zugriff 23.04.2015).

Avril, François: Un chef-d'œuvre de l'enluminure sous la règne de Jean le Bon. La Bible moralisée français 167 de la Bibliothèque nationale, in: Monuments et mémoires de la Fondation Piot, 58.1972, 91-125.

Biermann, Alfons W.: Die Miniaturenhandschriften des Kardinals Albrecht von Brandenburg (1514-1545), in: Aachener Kunstblätter, 46.1975, 15-310.

Brinkmann, Bodo: The Hastings Hours and the master of 1499, in: The British Library Journal, 14.1988, 90-106.

Buck, Stephanie: Die niederländischen Zeichnungen des 15. Jahrhunderts im Berliner Kupferstichkabinett. Kritischer Katalog, Turnhout 2001.

80 Judith Testa hat als Erste auf eine mögliche Verbindung der Miniatur mit dem Diptychon des Meisters von 1499 hingewiesen, cf. Testa 1984, 195. 
Buck, Stephanie: On relationships between Netherlandish drawing and manuscript illumination in the fifteenth century, in: Flemish manuscript painting in context. Recent research, hg. v. Elisabeth Morrison und Thomas Kren, Los Angeles 2006, 103-116.

[Cennini, Cennino:] Das Buch von der Kunst oder Tractat der Malerei des Cennino Cennini da Colle di Valdelsa [ca. 1300], übers. v. Albert Ilg (Quellenschriften für Kunstgeschichte und Kunsttechnik des Mittelalters und der Renaissance, 1), Wien 1871.

[Cennini, Cennino:] Il Libro dell'Arte, o Trattato della Pittura [ca. 1300] di Cennino Cennini da Colle di Valdelsa, hg. v. Gaetano u. Carlo Milanesi, Florenz 1859.

Chavannes-Mazel, Claudine Albertine: The Miroir Historial of Jean Le Bon. The Leiden manuscript and its related copies, Diss., Rijksuniversiteit te Leiden, Leiden 1988.

Coggiola, Giulio: Das Breviarium Grimani in der St. Markus-Bibliothek in Venedig. Seine Geschichte und seine Kunst, Textband, Leiden [u. a.] 1908.

Colenbrander, Herman Th.: The Limbourg brothers, the miniaturists of the Très Riches Heures du Duc de Berry? in: Masters and miniatures. Proceedings of the congress on medieval manuscript illumination in the Northern Netherlands, hg. v. Koert Van der Horst, Johann Klamt und Johann Christian (Studies and facsimiles of Netherlandish illuminated manuscripts, 3), Doornspijk 1991, 109-116.

Cupperi, Walter: Introduction. Never identical, multiples in pre-modern art?, in: Multiples in pre-modern art, hg. v. Walter Cupperi, Zürich [u. a.] 2014, 7-30.

De Winter, Patrick M.: A book of hours of Queen Isabel la Católica, in: The bulletin of the Cleveland Museum of Art, 68.1981 (10), 342-427.

Deleuze, Gilles: Différence et répétition, Paris 1968.

Deleuze, Gilles: Differenz und Wiederholung, übers. v. Joseph Vogl, München 1992.

Delisle, Léopold, Livres d'images destinés à l'instructions religieuse et aux exercieces de piété des laïques, in: Histoire littéraire de la France, 31.1890, 213-285.

Dijkstra, Jeltje: Origineel en kopie. Een onderzoek naar de navolging van de Meester van Flémalle en Rogier van der Weyden, Amsterdam 1990.

Dückers, Rob: „Im Anfang .... Die Stellung der Bible moralisée im Werk der Gebrüder Limburg, in: Ausst.-Kat. Die Brüder van Limburg. Nijmegener Meister am französischen Hof (1400-1416) (Nijmegen, Museum Het Valkhof 2005), hg. v. Rob Dückers und Pieter Roelofs, Stuttgart [u.a.] 2005, 84-95.

Durrieu, Paul: Manuscrits de luxe exécutés pour des princes et des grands seigneurs français (notes et monographies), in: Le Manuscrit, 2.1895, 1-5, 17-21, 34 f., 49-54, 65 f., 81-87, 97-103, 113-122, 129-135, 145-149, 162-168, 177-181.

Durrieu, Paul: Les Très Riches Heures du duc de Berry conservées à Chantilly, au Musée Condé, et le bréviaire Grimani, in: Bibliothèque de l'école des chartes, 64.1903 (1), 321-328.

Eeckhout, Paul: Les trois diptyques du Maître de 1499, in: Bulletin Musées royaux des Beaux-Arts, 34/37.1985/1988, 49-62.

Faries, Molly: Making and marketing. Studies of the painting process, in: Making and marketing. Studies of the painting process in fifteenth- and sixteenth-century Netherlandish workshops, hg. v. Molly Faries, Turnhout 2006, 1-14.

Gorissen, Friedrich: Jan Maelwael und die Brüder Limburg. Eine Nimweger Künstlerfamilie um die Wende des 14. Jhs., in: Gelre. Vereeniging tot beoefening van Geldersche Geschiedenis, Oudheidkunde en Recht, Bijdragen en Mededelingen, 54.1954, 153-221.

Grote, Andreas: Breviarium Grimani, Faksimileausgabe der Miniaturen und Kommentar, Berlin 1973.

Heyder, Joris Corin: Corporate design made in Ghent-Bruges? On the extensive reuse of patterns in late medieval Flemish illuminated manuscripts, in: The use of models in medieval book painting, hg. v. Monika Müller, Newcastle upon Tyne 2014, 167-201.

Heyder, Joris Corin: Re-Inventing traditions? Preliminary thoughts on the transmission of artistic patterns in late medieval manuscript illumination, in: Re-inventing traditions. On the transmission of artistic patterns in late medieval manuscript illumination, hg. v. Joris Corin Heyder und Christine Seidel (Zivilisationen und Geschichte, 34), Frankfurt a. M. [u. a.] 2015, 13-27.

Heyer, Stefan: Deleuze und Guattaris Kunstkonzept. Ein Wegweiser durch Tausend Plateaus, Wien 2001. 
Hilger, Wolfgang: Das Ältere Gebetbuch Maximilians I. Vollständige Faksimile-Ausgabe im Originalformat des Codex Vindobonensis 1907 der Österreichischen Nationalbibliothek (Codices selecti, Phototypice Impressi, 39), Graz 1973.

Hoffmann, Volker: Masaccios Trinitätsfresko. Die Perspektivkonstruktion und ihr Entwurfsverfahren, in: Mitteilungen des Kunsthistorischen Institutes in Florenz, 40.1996, 43-77.

Kemperdick, Stephan: Von der Vorlage zum Kunstwerk. Rogier van der Weydens ,Große Kreuzabnahme', in: Original - Kopie - Zitat, hg. v. Wolfgang Augustyn und Ulrich Söding (Veröffentlichungen des Zentralinstituts für Kunstgeschichte in München, 26), Passau 2010, 207-230.

Kimmerle, Heinz: Philosophien der Differenz: eine Einführung (Schriften zur Philosophie der Differenz, 9), Würzburg 2000.

Koller, Manfred: Das Staffeleibild der Neuzeit, in: Reclams Handbuch der künstlerischen Techniken. Farbmittel, Buchmalerei, Tafel- und Leinwandbild, 1. Bd., Stuttgart 1984, 261-434.

König, Eberhard: Französische Buchmalerei um 1450, Berlin 1982.

König, Eberhard: Die Bible moralisée der Limburgs. Von den Brüdern Limburg zu Georges Trubert, Kommentarband zur Faksimile-Ausgabe, Valencia 2010, 261-305 (sowie spanische Fassung, ebd., 15-59; italienische Fassung, ebd., 89-141; englische Fassung, ebd., 171-223).

König, Eberhard / Heyder, Joris Corin, Das Breviarium Grimani, Simbach am Inn 2016.

Krieger, Michaela: Gerard Horenbout und der Meister Jakobs IV. von Schottland. Stilkritische Überlegungen zur flämischen Buchmalerei, Wien 2012.

Lieftinck, Gerard Isaac: De Meester van Maria van Bourgondië en Rooclooster bij Brussel, in: Bulletin \& Nieuws-Bulletin Koninklijke Nederlandse Oudheidkundige Bond, 17.1964, 255-296.

Lieftinck, Gerard Isaac: Boekverluchters uit de omgeving van Maria van Bourgondie, c.1475 - c.1485, Brüssel 1969.

Maier, Ingo: The Barberinus and Munich codices of the notitia dignitatum omnium, in: Latomus, 28.1969, 960-1035.

Marciari, John / Verstegen, Ian: „Grande quanto l'opera“. Size and scale in Barocci's drawings, in: Master Drawings, 46.2003 (3), 291-321.

Martens, Maximiliaan: La production de masse d'oeuvres d'art. Un concept erroné, le cas de la peinture anversoise durant la première moitié du XVIe siècle, in: L'art multiplié. Production de masse, en série, pour le marché dans les arts entre Moyen Âge et Renaissance, hg. v. Michele Tomasi (Études lausannoises d'historie de l'art, 11), Viella 2011, 105-114.

McMahon, Melissa: Difference, repetition, in: Gilles Deleuze: Key Concepts, hg. v. Charles J. Stivale, Chesham 2007, 42-52.

Meder, Joseph: Die Handzeichnung, ihre Technik und Entwicklung, Wien 1923.

Mensger, Ariane: Jan van Eyck, Belgarum Splendor, und der Anfang einer niederländischen Geschichte der Kunst, in: Pantheon, 58.2000, 44-51.

Mensger, Ariane: Die exakte Kopie. Oder: die Geburt des Künstlers im Zeitalter seiner Reproduzierbarkeit, in: Nederlands Kunsthistorisch Jaarboek, 59.2009/2010, 194-221.

Münker, Stefan/Roesler, Alexander: Poststrukturalismus, Stuttgart [u. a.] 2000.

Mund, Hélène: Copie et traditionalisme dans la peinture flamande des XVe et XVIe siècles, (unveröffentlichte) Diss., Faculté de philosophie et lettres, archéologie et histoire de l'art, Université catholique de Louvain, Löwen 1992.

Nash, Susie: Imitation, invention, or good business sense? The use of drawings in a group of fifteenth-century French books of hours, in: Drawing 1400-1600. Invention and innovation, hg. v. Stuart Currie, Susie Nash und Julia Watson, Aldershot 1998, 12-25.

Pächt, Otto: The master of Mary of Burgundy, London 1948.

Pächt, Otto / Thoss, Dagmar: Flämische Schule II (Die illuminierten Handschriften und Inkunabeln der Österreichischen Nationalbibliothek, 7), Wien 1990.

Panfosky, Erwin: Die altniederländische Malerei. Ihr Ursprung und Wesen (Übersetzung der Originalausgabe "Early Netherlandish Painting: its origins and character", Cambridge/Mass. 1953), hg. v. Jochen Sander und Stephan Kemperdick, 2 Bde., Köln 2001.

Parr, Adrian (Hg.): The Deleuze Dictionary. Revised Edition, Edinburgh 2013.

Powell, Amy: A point "ceaselessly pushed back". The origin of early Netherlandish painting, in: The art bulletin, 88.2006, 707-728. 
Remak-Honnef, Elisabeth / Hauke, Hermann: Katalog der lateinischen Handschriften der Bayerischen Staatsbibliothek München. Die Handschriften der ehem. Mannheimer Hofbibliothek: Clm 10001-10930, ausgenommen die Codices Lullani (Clm 10493-10658) und die Sammlung Camerius (Clm 10351-10431) (Catalogus codicum manu scriptorum Bibliothecae Monacensis, T.4, Ser. nov. Ps. 1), Wiesbaden 1991.

Schenk zu Schweinsberg, Eberhard: Das Gebetbuch für Graf Engelbert II. von Nassau und seine Meister, in: Nassauische Annalen. Jahrbuch des Vereins für Nassauische Altertumskunde und Geschichtsforschung, 68.1975, 139-157.

Steinmann, Martin: Handschriften im Mittelalter. Eine Quellensammlung, Basel 2013.

Sterling, Charles: La peinture médiévale à Paris. 1300-1500, 2 Bde., Paris 1987.

Tarde, Gabriel: Les lois de l'imitation, Paris 1890.

Testa, Judith Anne: The Beatty Rosarium reconstructed. A manuscript with excised miniatures by Simon Bening, in: Oud Holland, 98.1984 (4), 189-236.

Thimann, Michael: „Idea" und "Conterfei“. Künstlerisches und wissenschaftliches Zeichnen in der Frühen Neuzeit, in: Disegno. Der Zeichner im Bild der Frühen Neuzeit, hg. v. Hein-Th. Schulze Altcappenberg und Michael Thimann, Berlin 2007, 15-30.

Turner, Derek Howard: The Hastings Hours. A $15^{\text {th }}$-century Flemish book of hours made for William, Lord Hastings, now in the British Library, London, New York 1983.

Van Buren, Anne H.: The master of Mary of Burgundy and his colleagues. The state of research and questions of method, in: Zeitschrift für Kunstgeschichte, 38.1975 (3/4), 286-309.

Van den Brink, Peter: L'art de la copie. Le pourquoi et le comment de l'exécution de copies aux Pays-Bas aux XVIe et XVIIe siècles, in: Ausst.-Kat. L'entreprise Brueghel (Maastricht, Bonnefantenmuseum 2001; Brüssel, Musée royaux des beaux-arts 2002), hg. v. Dominique Allart und Peter Van den Brink, Gent 2001, 12-43.

Van Grieken, Joris: La copie mise en contexte. Enquète sur les copies et les gravures d'après les maîtres anciens des Pays-Bas, à partir de leur reception dans la deuxième moitié du XVIe siècle, in: L'estampe un art multiple à la portée de tous?, hg. v. Sophie Raux, Nicolas Surlapierre und Dominique Tonneau-Ryckelynck, Lille 2008, 29-45.

Vrij, Marc Rudolf: Marcellus Coffermans, Amsterdam 2003.

Wijsman, Hanno: Luxury bound. Illustrated manuscript production and noble and princely book ownership in the Burgundian Netherlands (1400-1550) (Burgundica, 16), Turnhout 2010. 